\title{
Airborne observations and simulations of three-dimensional radiative interactions between Arctic boundary layer clouds and ice floes
}

\author{
M. Schäfer ${ }^{1}$, E. Bierwirth ${ }^{1, a}$, A. Ehrlich ${ }^{1}$, E. Jäkel ${ }^{1}$, and M. Wendisch ${ }^{1}$ \\ ${ }^{1}$ Leipzig Institute for Meteorology, University of Leipzig, Leipzig, Germany \\ ${ }^{a}$ now at: PIER-ELECTRONIC GmbH, Nassaustr. 33-35, 65719 Hofheim-Wallau, Germany
}

Correspondence to: M. Schäfer (michael.schaefer@uni-leipzig.de)

Received: 12 December 2014 - Published in Atmos. Chem. Phys. Discuss.: 19 January 2015

Revised: 5 June 2015 - Accepted: 7 July 2015 - Published: 23 July 2015

\begin{abstract}
Based on airborne spectral imaging observations, three-dimensional (3-D) radiative effects between Arctic boundary layer clouds and highly variable Arctic surfaces were identified and quantified. A method is presented to discriminate between sea ice and open water under cloudy conditions based on airborne nadir reflectivity $\gamma_{\lambda}$ measurements in the visible spectral range. In cloudy cases the transition of $\gamma_{\lambda}$ from open water to sea ice is not instantaneous but horizontally smoothed. In general, clouds reduce $\gamma_{\lambda}$ above bright surfaces in the vicinity of open water, while $\gamma_{\lambda}$ above open sea is enhanced. With the help of observations and 3$D$ radiative transfer simulations, this effect was quantified to range between 0 and $2200 \mathrm{~m}$ distance to the sea ice edge (for a dark-ocean albedo of $\alpha_{\text {water }}=0.042$ and a sea-ice albedo of $\alpha_{\text {ice }}=0.91$ at $645 \mathrm{~nm}$ wavelength). The affected distance $\Delta L$ was found to depend on both cloud and sea ice properties. For a low-level cloud at 0-200 $\mathrm{m}$ altitude, as observed during the Arctic field campaign VERtical Distribution of Ice in Arctic clouds (VERDI) in 2012, an increase in the cloud optical thickness $\tau$ from 1 to 10 leads to a decrease in $\Delta L$ from 600 to $250 \mathrm{~m}$. An increase in the cloud base altitude or cloud geometrical thickness results in an increase in $\Delta L$; for $\tau=1 / 10$ $\Delta L=2200 \mathrm{~m} / 1250 \mathrm{~m}$ in case of a cloud at $500-1000 \mathrm{~m}$ altitude. To quantify the effect for different shapes and sizes of ice floes, radiative transfer simulations were performed with various albedo fields (infinitely long straight ice edge, circular ice floes, squares, realistic ice floe field). The simulations show that $\Delta L$ increases with increasing radius of the ice floe and reaches maximum values for ice floes with radii larger than $6 \mathrm{~km}$ (500-1000 m cloud altitude), which matches the results found for an infinitely long, straight ice edge.
\end{abstract}

Furthermore, the influence of these 3-D radiative effects on the retrieved cloud optical properties was investigated. The enhanced brightness of a dark pixel next to an ice edge results in uncertainties of up to 90 and $30 \%$ in retrievals of $\tau$ and effective radius $r_{\text {eff }}$, respectively. With the help of $\Delta L$, an estimate of the distance to the ice edge is given, where the retrieval uncertainties due to 3-D radiative effects are negligible.

\section{Introduction}

As shown by observations and simulations, the Arctic climate changes faster and stronger than the global climate (e. g., Sanderson et al., 2011; Overland et al., 2011). Among others, Vavrus (2004) identified clouds as a major source of uncertainty in model predictions of the future Arctic climate. Therefore, understanding the effects of clouds in the Arctic climate system is of utmost importance. Depending on the time of year and their altitude, Arctic clouds may exert either a net warming or cooling effect. However, the low sun in summer combined with a usually high surface albedo lead to a dominance of the terrestrial (infrared) radiative warming of low clouds (Intrieri et al., 2002b; Wendisch et al., 2013). For the solar wavelength range, the surface albedo (sea ice coverage) is a major parameter determining whether a change of cloud amount in future climate is associated with a warming or cooling effect.

While Arctic stratus often shows a horizontally homogeneous structure, both in macrophysical (cloud base and top 
altitude) and microphysical properties, sea ice is often characterized by a more heterogeneous horizontal distribution. Tsay and Jayaweera (1984) showed that Arctic stratus has a considerable horizontal homogeneity of cloud morphology, droplet diameter, concentration, and liquid water content, except for the cloud top layer. Here, mixing results in small-scale inhomogeneities identified by Lawson et al. (2001) and Klingebiel et al. (2015): bi-modal cloud particle size distributions at cloud top, while mono-modal distributions dominate the lower cloud layers representative for the adiabatic and homogeneous character of the clouds. In contrast, sea ice has irregular top and bottom surfaces and is broken into distinct pieces, called floes (Rothrock and Thorndike, 1984). Openings in the ice surface (cracks, leads, and polynias) are often present especially in the transition zone between sea ice and open water, often accompanied by fields of scattered ice floes. The albedo contrast in such areas is the highest we can observe on Earth. For visible wavelengths, the albedo of open water is low $(0.042$ at $645 \mathrm{~nm}$; Bowker et al., 1985), while that of ice-/snow-covered ocean is high (0.91 at $645 \mathrm{~nm}$; Bowker et al., 1985). These differences significantly decrease in the near-infrared wavelength range $\left(\alpha_{\text {water }}=0.01\right.$ and $\alpha_{\text {snow }}=0.04$ at $\lambda=1.6 \mu \mathrm{m}$ wavelength; Bowker et al., 1985), but still slightly alter the radiative transfer.

Using Advanced Very High Resolution Radiometer (AVHRR) data from the polar-orbiting satellites NOAA-10 and NOAA-11, Lindsay and Rothrock (1994) analyzed the albedos of 145 different $200 \mathrm{~km}^{2}$ cells in the Arctic. The mean values for the cloud-free portions of individual cells range from 0.18 to 0.91 and were found to be highly variable at monthly and annual time scales (Lindsay and Rothrock, 1994).

As demonstrated by Bierwirth et al. (2013), airborne remote sensing using spectral imaging sensors is one promising method to characterize small scale inhomogeneities of clouds in the spatial range below $5 \mathrm{~m}$. For airborne imaging spectrometer measurements over dark ocean surfaces, Bierwirth et al. (2013) introduced a novel five-wavelength cloud retrieval procedure that is based on the classic twowavelength cloud retrieval by Nakajima and King (1990) and follows the multi-wavelength approach by Coddington et al. (2012) and King and Vaughan (2012). For airborne measurements performed during the international field campaign SoRPIC (Solar Radiation and Phase discrimination of ArctIc Clouds), Bierwirth et al. (2013) showed that accurate retrieval results can be obtained for the cloud optical thickness $\tau$. However, due to the limitation of the instrument to wavelengths below $1000 \mathrm{~nm}$ a retrieval of $r_{\text {eff }}$ was not feasible. Also, the application of the retrieval was restricted to areas of open water.

A highly variable Arctic surface albedo as observed during the VERDI campaign complicates the cloud retrieval introduced by Bierwirth et al. (2013). In fact, retrievals of cloud microphysical and optical properties using only visible wavelengths are strongly biased by a bright surface (Platnick et al., 2001, 2004; Platnick and King, 2003; Krijger et al., 2011). To overcome this limitation, near-infrared channels are introduced in the retrieval algorithms instead of the visible channel used over dark surfaces. E.g., for MODIS the $1.6 \mu \mathrm{m}$ band reflectance is applied as a surrogate for the traditional non-absorbing band in conjunction with a stronger absorbing 2.1 or $3.7 \mu \mathrm{m}$ band (Platnick et al., 2001, 2004; Platnick and King, 2003). However, an accurate separation between sea ice and open water needs to be performed before the retrieval algorithms are applied. Operational algorithms such as that for MODIS use NOAA's (National Oceanic and Atmospheric Administration) microwave-derived daily $0.25^{\circ}$ Near Real-Time Ice and Snow Extent (NISE) data set (Armstrong and Brodzik, 2001; Platnick and King, 2003) to identify snow- or ice-covered scenes. For the typical scale of ice floes which can be observed from aircraft, this pixel size is not sufficient. Algorithms using the resolution of the radiance measurements have to be applied.

However, even when ice and ice-free areas are perfectly separated by the retrieval algorithms, 3-D radiative effects may still affect the cloud retrieval over ice-free pixels close to the ice edge. With respect to the large temporal and spatial variability of the Arctic surface albedo as described by Lindsay and Rothrock (1994), the investigation of the 3-D effects becomes even more important. Lyapustin (2001) and Lyapustin and Kaufman (2001) investigated the impact of the strong contrast of the surface albedo between open sea and adjacent sea-ice or snow on the retrieval of Arctic cloud properties. Adjacency effects were found to reduce the apparent surface contrast by decreasing the top-of-the-atmosphere $\gamma_{\lambda}$ over bright pixels and increasing the brightness of dark pixels, which becomes important for land remote-sensing applications developed for usage with both dark or bright targets.

Within the present study, the focus lies on those 3-D radiative effects that are related to the horizontal photon transport between cloud and surface due to isotropic reflection of the incident radiation on the bright sea ice. The goal is to quantify the magnitude and horizontal extent of those 3-D effects as well as their influence on cloud retrievals from the visible wavelength range with a high spatial resolution. In reality, such surface 3-D radiative effects will be combined with cloud 3-D radiative effects due to cloud inhomogeneities. However, in the case of Arctic stratus, the individual cloud 3 -D effect is of minor importance. For a solar zenith angle $(\Theta)$ of $45^{\circ}$, Zinner et al. (2010) found that the remote sensing of stratocumulus was not biased by 3-D effects, while that of scattered cumulus was sensitive to horizontal heterogeneities. This leads to the assumption that retrievals of cloud microphysical and optical properties can be treated by 1-D simulations if the distance to ice-open water boundaries is sufficiently large. However, measurements in Arctic regions are often performed for solar zenith angles larger than $45^{\circ}$. In such cases, 3-D radiative effects generated by the cloud structures become important. Using plane-parallel 1-D simu- 
lations of clouds, Loeb and Davis (1996) stated that the cloud optical thickness shows a systematic shift towards larger values with increasing solar zenith angle. This dependence is still weak $(\leq 10 \%)$ for thin clouds $(\tau \leq 6)$ and $\Theta \leq 63^{\circ}$. Grosvenor and Wood (2014) confirmed this statement. They investigated MODIS satellite retrieval biases of $\tau$ and stated that $\tau$ is fairly constant between $\Theta=50^{\circ}$ and $\approx 65-70^{\circ}$, but then increases rapidly with an increase of over $70 \%$ between the lowest and highest $\Theta$.

The individual 3-D effect of heterogeneous surfaces in cloud-free situations was investigated by Jäkel et al. (2013). They quantified the effect of local surface-albedo heterogeneity and aerosol parameters on the retrieved areaaveraged surface albedo from airborne upward and downward irradiance measurements. For adjacent land and sea, Jäkel et al. (2013) defined a critical distance $d_{\mathrm{c}}$ at which the retrieved area-averaged surface albedo deviates by $10 \%$ or less from the given local surface albedo. It was found that $d_{\mathrm{c}}$ ranges in the order of $2.4 \mathrm{~km}$ for a flight altitude of $2 \mathrm{~km}$ and is larger for albedo fields with higher surface albedo contrast. In the case of clouds with an optical thickness larger than that of aerosol particles, this effect is expected to increase significantly.

In Sect. 2 we present airborne observations of $\gamma_{\lambda}$ derived from imaging spectrometer measurements of upwelling radiance $I_{\lambda}^{\uparrow}$ and spectrometer measurements of downwelling irradiance $F_{\lambda}^{\downarrow}$. A robust algorithm separating sea-ice and openwater surfaces under cloud cover is introduced in Sect. 3 and applied to the measurements. Observations and simulations of the 3-D radiative effects are analyzed in Sect. 4. Similar to Jäkel et al. (2013), a critical distance from the ice edge is defined to quantify the horizontal range of the effects. For the model simulations, idealized surface albedo fields (infinitely long straight ice edge, circular ice floes of different sizes, groups of ice floes) are generated and investigated for cases of clear sky and for $\tau=1 / 5 / 10$. Variations in cloud altitude, cloud geometrical thickness, $r_{\text {eff }}$, and surface albedo are investigated to characterize how strong these parameters influence the magnitude and distance of the $\gamma_{\lambda}$ transition from high to low values. In Sect. 5 we investigate how these 3-D radiative effects bias 1-D retrievals of $\tau$ and $r_{\text {eff. }}$.

\section{Airborne measurements of spectral reflectivity $\gamma_{\lambda}$}

The measurements used in this study were taken during the international Arctic field campaign VERDI, which took place in Inuvik, Northwest Territories, Canada, in April and May 2012. The instruments were installed on Polar 5, an aircraft used for scientific research by the Alfred Wegener Institute Helmholtz Centre for Polar and Marine Research (AWI), Bremerhaven. During VERDI, the Polar 5 was operated out of the Inuvik Mike Zubko Airport (YEV). Most flights were performed over the Beaufort Sea, partly covered by sea ice interspersed with open leads and polynias which grew bigger towards the end of the campaign.

The measurement strategy during VERDI was aimed at combining remote sensing and in situ cloud observations. Therefore, the same clouds were subsequently sampled by a set of in situ (see Klingebiel et al., 2015) and remotesensing instruments on board of Polar 5. The aircraft was equipped with an active and several passive remote-sensing systems. The active system was the Airborne Mobile Aerosol Lidar (AMALi; Stachlewska et al., 2010). It was operated in nadir viewing direction at $532 \mathrm{~nm}$ wavelength. Passive radiation measurements were carried out with the imaging spectrometer AisaEAGLE (manufactured by Specim Ltd. in Oulu, Finland; Schäfer et al., 2013). To analyze the 3-D radiative effects of ice edges in a cloudy atmosphere, we focus on measurements by this instrument. With 1024 spatial pixels, the single-line sensor provides a sufficiently high horizontal resolution to observe ice edges in detail. The flight altitude during the remote sensing legs was about $3 \mathrm{~km}$ above ground which is about $2 \mathrm{~km}$ above cloud top for typical boundary layer clouds with cloud top altitudes at about $1 \mathrm{~km}$. For this geometry, the width of one AisaEAGLE pixel at cloud top is $3.5 \mathrm{~m}$ and the length is $4.2 \mathrm{~m}$ at an exposure time of $10 \mathrm{~ms}$ and a flight speed of $65 \mathrm{~m} \mathrm{~s}^{-1}$. Each spatial pixel consists of 488 spectral pixels to detect spectra of radiance in the wavelength range from 400 to $970 \mathrm{~nm}$ with $1.25 \mathrm{~nm}$ full width at half maximum (FWHM). AisaEAGLE converts the detected photon counts into digitalized 12bit numbers. By applying a spectral radiometric calibration, those numbers are transformed into radiances. The calibration, data handling, and necessary corrections are described by Schäfer et al. (2013). For radiance measurements, Schäfer et al. (2013) estimated an uncertainty of $\pm 6 \%$. Assuming a fixed $r_{\text {eff }}$, those detected spectra of radiance can then be used to retrieve $\tau$.

Further passive radiation measurements were carried out with the Spectral Modular Airborne Radiation measurement system (SMART-Albedometer; Wendisch et al., 2001), initially designed for albedo measurements, and a Sun tracking photometer. The SMART-Albedometer is horizontally stabilized and measures up-/downwelling spectral radiance $I_{\lambda}$ and irradiance $F_{\lambda}(\lambda=350-2100,2-16 \mathrm{~nm}$ FWHM), while the Sun photometer covers aerosol optical thickness between $\lambda=367-1026 \mathrm{~nm}$. The configuration was similar to that during the aircraft campaign SoRPIC described by Bierwirth et al. (2013). Additionally, dropsondes were used at selected waypoints to sample profiles of meteorological parameters (air pressure, air temperature, relative humidity) over the whole distance between the ground and the aircraft. For a more detailed description of the airborne instruments installed on Polar 5, see Bierwirth et al. (2013), Klingebiel et al. (2015) and Wendisch and Brenguier (2013).

In this study, the data from the AMALi and the dropsondes were used to determine the cloud-top altitude and geometrical thickness, whereas the data from the SMART- 


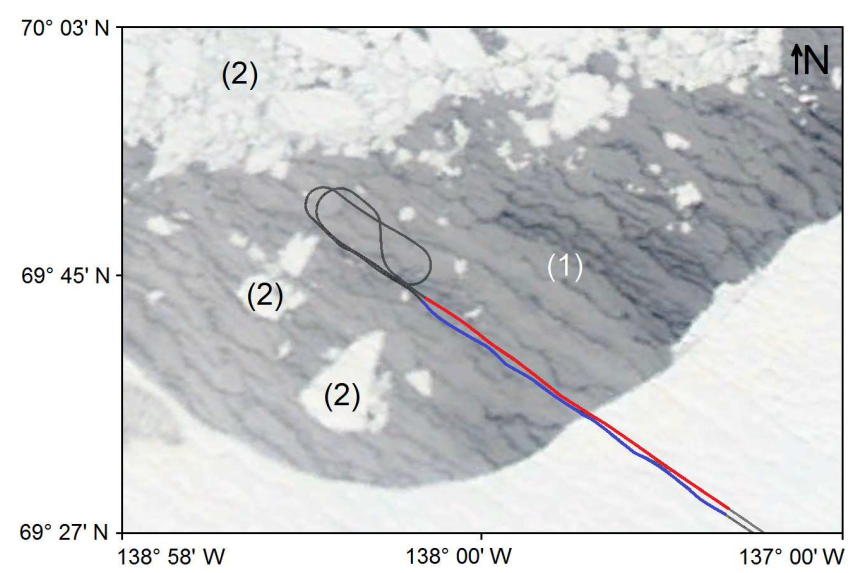

Figure 1. VERDI flight track and true-color MODIS image (Aqua; $250 \mathrm{~m}$ resolution) from 17 May 2012. Numbers (1) and (2) label open ocean and sea ice, respectively.

Albedometer were used to verify and validate the $I_{\lambda}^{\uparrow}$ measurements of AisaEAGLE. Furthermore, the SMARTAlbedometer measurements of the downwelling irradiance $F_{\lambda}^{\downarrow}$ are used to transform the AisaEAGLE radiance $I_{\lambda}^{\uparrow}$ into the nadir reflectivity $\gamma_{\lambda}$, which is derived by:

$\gamma_{\lambda}\left(\tau, r_{\mathrm{eff}}\right)=\frac{\pi \cdot I_{\lambda}^{\uparrow}\left(\tau, r_{\mathrm{eff}}\right)}{F_{\lambda}^{\downarrow}\left(\tau, r_{\mathrm{eff}}\right)}$,

and is a function of $r_{\text {eff }}$ and $\tau$. The $\gamma_{\lambda}$ presented in this paper are calculated at a wavelength of $\lambda=645 \mathrm{~nm}$, where scattering is dominant and shows a strong sensitivity to $\tau$ (Werner et al., 2013).

During all 15 flights of VERDI, 130 recordings $(25 \mathrm{~h}, 11 \mathrm{~min}, 29 \mathrm{~s})$ of reflected radiance from cloud-top and surface were collected with AisaEAGLE. $78 \%$ of the observation time was spent above clouds. However, for $86 \%$ of the cloud observations a cloud retrieval as described by Bierwirth et al. (2013) could not be applied as the surface albedo did not fulfill the constraint of being relatively dark. Either snow-covered ice almost eliminated the contrast between cloud and surface, or a mixture of ice and open water made a cloud retrieval following the strategy from Bierwirth et al. (2013) impossible. The latter occurred in $42 \%$ of all observations and is analyzed in this paper in more detail to quantify the degree to which cloud retrievals are biased above such heterogeneous surfaces.

\section{Identification of ice and open water}

A typical scene showing a mixture of sea ice and open ocean surfaces covered by an optically thin stratus is shown in Fig. 1. Using only the visible wavelength channels of the image, no visible contrast between sea ice and cloud remains. This is why near-infrared channels are applied in MODIS

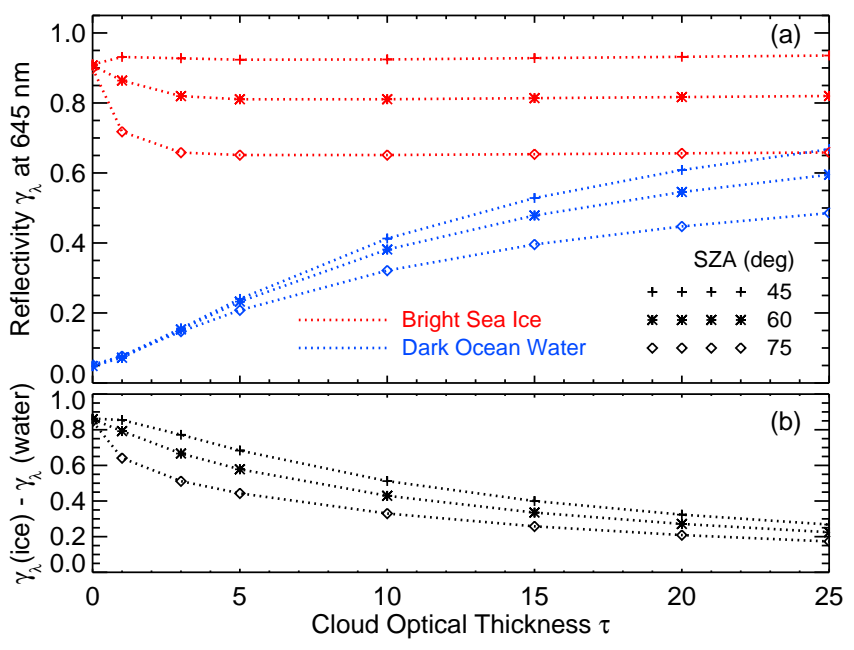

Figure 2. (a) Simulated $\gamma_{\lambda}$ at $645 \mathrm{~nm}$ calculated for different $\tau$ ranging from 0 to 25 and cloud particles with a fixed $r_{\text {eff }}$ of $15 \mu \mathrm{m}$. The calculations were performed for different $\Theta$ of 45,60 , and $75^{\circ}$ over a highly reflecting ice surface and a dark ocean surface. (b) Difference between the simulated $\gamma_{\lambda}$ over bright sea ice surface and dark ocean surface from (a).

cloud retrievals over sea ice. However, with AisaEAGLE the observations are limited to wavelength below $1000 \mathrm{~nm}$, where the contrast is weak (compare Fig. 4) and a retrieval of $\tau$ is not possible in those areas; it can only be performed above water surfaces.

The limitation of AisaEAGLE in case of bright surfaces is illustrated in Fig. 2 showing the calculated $\gamma_{\lambda}$ at $645 \mathrm{~nm}$ for clouds with different values of $\tau$ over a dark ocean surface (blue lines) and a bright sea-ice surface (red lines). The calculations were performed for different solar zenith angles $\Theta$ of $45^{\circ}, 60^{\circ}$, and $75^{\circ}$ (the range during VERDI measurements).

The upper panel shows that the separation of $\gamma_{\lambda}$ for clouds of different $\tau$ above sea ice are not significant and far below the measurement uncertainties of most optical sensors. This illustrates that no physical information on the cloud optical thickness is given by $\gamma_{\lambda}$ at this visible wavelength of $645 \mathrm{~nm}$. Therefore, a retrieval of $\tau$ based on $645 \mathrm{~nm}$ reflectivity is not possible. For the same clouds placed above a dark ocean surface, $\gamma_{\lambda}$ is a strong function of $\tau$, which is the basis of the cloud retrieval following the method by Nakajima and King (1990), Bierwirth et al. (2013), and Werner et al. (2013).

In order to select the dark-surface pixels for which a cloud retrieval can be attempted, a sea-ice mask has to be derived. Figure 2 clearly shows that even for optically thick clouds $\gamma_{\lambda}$ is significantly larger ( $\geq 25 \%$ at $\tau=25$ and $\Theta=60^{\circ}$ ) above bright sea ice than over a dark ocean surface. This gap can be used as a threshold to distinguish between measurements of clouds above the dark ocean surface and a bright sea ice surface. To define this threshold, it has to be considered that the differences between $\gamma_{\lambda}$ measured above a dark ocean surface 


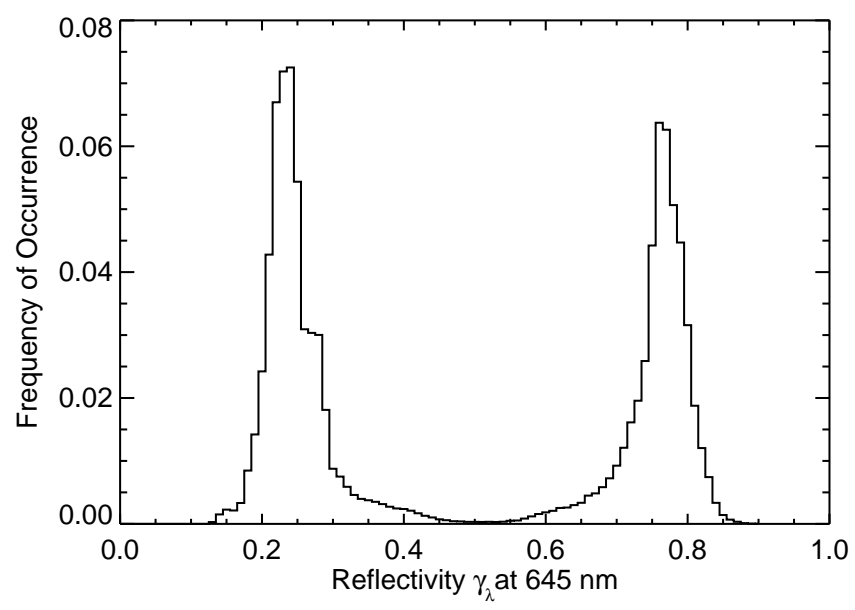

Figure 3. Fraction of occurrence of the measured $\gamma_{\lambda}$ at $645 \mathrm{~nm}$, given in the example of Fig. $4 \mathrm{a}$. The bin size is 0.01 .

or a bright sea ice surface is smaller for larger solar zenith angles and also decreases with increasing $\tau$ (lower panel of Fig. 2). However, the differences are still significant at large solar zenith angles of $\Theta=60^{\circ}$ and $\tau=25$ (lower panel). For VERDI, where $\Theta$ was in the range of 55 to $75^{\circ}$ for most of the observations, the particular threshold is defined as the center value between the two simulations:

$\gamma_{\lambda, \text { thresh }}=\frac{\gamma_{\lambda, \text { ice }}+\gamma_{\lambda, \text { water }}}{2}$.

To test this threshold, a section of a VERDI flight on 17 May 2012 (Fig. 1) was analyzed. The flight was divided into a remote-sensing leg A at $2920 \mathrm{~m}$ altitude (red in Fig. 1) and an in situ leg $B$ inside the cloud at $150 \mathrm{~m}$ altitude (blue in Fig. 1). The solar zenith angle was $\Theta=58^{\circ} . \tau$ was obtained from AisaEAGLE measurements above open water far from any ice edge using the retrieval method presented by Bierwirth et al. (2013). An average value of $\tau=5.3 \pm 0.5$ was derived, which agrees with the MODIS level-2 product showing values for $\tau$ between 0.02 and $15.5(\bar{\tau}=3.6 \pm 2.5)$ in the investigated area. In that case, the simulated $\gamma_{\lambda}$,ice $=0.8$ and $\gamma_{\lambda \text {,water }}=0.2$ from Fig. 2 give a threshold of $\gamma_{\lambda}$,thresh $=0.5$.

Figure 3 shows a histogram of the measured $\gamma_{\lambda}$ at $645 \mathrm{~nm}$ from leg A. There are two distinctly separated maxima which correspond to measurements above bright sea ice and dark ocean. Either the minimum between the two maxima or the mean of those two most frequent values of $\gamma_{\lambda}$ can be used as an alternative estimate of the threshold for the ice mask. In this particular case the threshold estimated from the frequency distribution is $\gamma \lambda$, thresh $=0.5$, which confirms the theoretical value derived from the radiative transfer simulations.

Using those methods to estimate the threshold, ice masks were created to identify measurements of clouds above sea ice for which the cloud retrieval by Bierwirth et al. (2013) cannot be applied. Figure 4 shows three examples of $\gamma_{\lambda}$ derived from the nadir radiance and zenith irradiance measure-

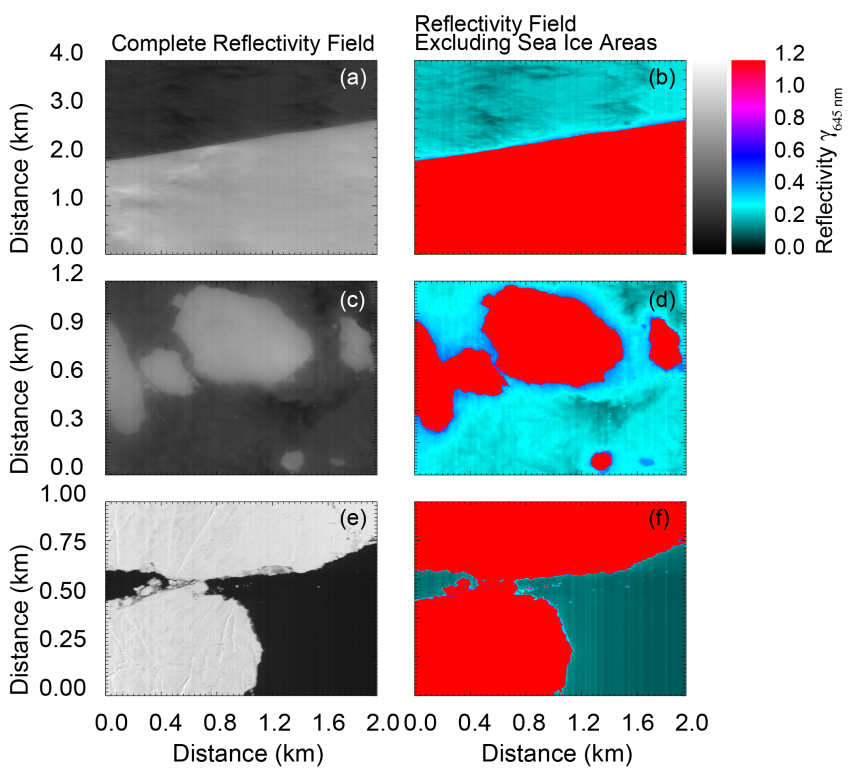

Figure 4. Left Side: Fields of $\gamma_{\lambda}$ at $645 \mathrm{~nm}$, measured with the imaging spectrometer AisaEAGLE. The measurements were performed on 17 May 2012 during the international field campaign VERDI. Right side: The same as on the left side in colour-scale and with ice mask overlay.

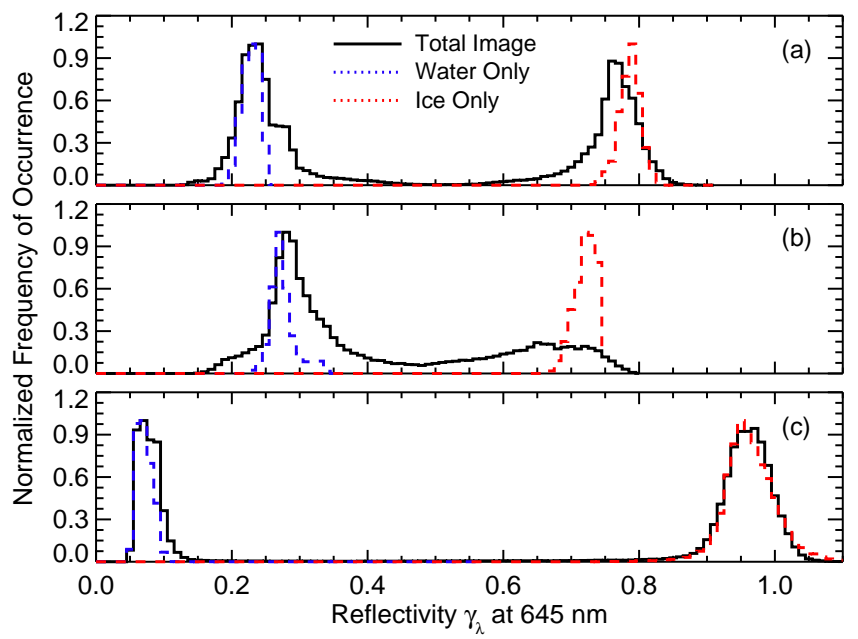

Figure 5. Normalized distributions of the frequency of occurrence of $\gamma_{\lambda}$ measured during the three cases presented in Fig. 4. Additionally included are the frequency distributions over sea ice and dark ocean water only.

ments. Figures $4 \mathrm{a}$ and $\mathrm{b}$ show a long ice edge, while Fig. $4 \mathrm{c}$ and $\mathrm{d}$ show an accumulated ice floe field, all observed on 17 May 2012 around 17:00 UTC $\left(\Theta=58^{\circ}\right)$. In both cases, a cloud layer was located between the ground and the aircraft. Figures $4 \mathrm{e}$ and $\mathrm{f}$ show ice floes without cloud cover, observed on 14 May 2012 at 21:00 UTC. The corresponding ice masks are shown on the right panel in Fig. 4. In those images, all pixels identified as sea ice are shown as red areas. 
Figure 5 shows the frequency distributions for the three cases presented in Fig. 4 (solid black lines). All frequency distributions are normalized so that the maximum is 1 . In each case, two maxima are separated by a distinct minimum which defines the ice/water threshold value. For the cloudy cases in Fig. 5a and b the dark-surface peak is broadened asymmetrically towards higher $\gamma_{\lambda}$ values, while the brightsurface peak is broadened towards lower $\gamma_{\lambda}$ values. For the clear-sky case in Fig. 5c, the peaks representing dark ocean and sea-ice surfaces are clearly separated.

To analyze the impact of the ice edge, frequency distributions for a selection of pixels far from the ice edge are included in Fig. 5 for each particular scene, separated into dark open-water pixels (dashed blue lines) and bright sea-ice pixels (dashed red lines). In Fig. 5a and b, the peaks of the selective frequency distributions are much sharper than the original peaks. For the clear-sky case in Fig. 5c, these selective frequency distributions are almost congruent with the single peaks of the entire frequency distribution. This means that in this case the ice edge has no impact on $\gamma_{\lambda}$ of adjacent pixels, or in other words, there is no significant horizontal photon transport. Between the remote-selected pixels and the ice edge there are many pixels where $\gamma_{\lambda}$ is enhanced (over open water) or reduced (over sea ice) compared to the values at the remote pixels. This particular enhancement and reduction of the measured $\gamma_{\lambda}$ is related to 3-D radiative effects in clouds and the reflection between clouds and the surface. This influence of the ice edge on the pixels' reflectivity will significantly influence cloud retrievals based on 1-D simulations in such scenes. In the following, those 3-D effects are investigated at first only by analyzing $\gamma_{\lambda}$ and then with respect to the retrieved cloud optical properties. To characterize the magnitude of the enhancement and reduction of the measured $\gamma_{\lambda}$, 3 -D radiative transfer simulations are performed in Sect. 4 and are used to identify the most important parameters that control this 3-D effect. Afterwards, a 1-D cloud retrieval is performed in Sect. 5 to quantify the influence of the 3-D effects on the retrieved $\tau$ and $r_{\text {eff. }}$.

\section{3-D radiative interactions between heterogeneous surfaces and clouds}

\subsection{Measurements from VERDI}

Figure 6 illustrates the measured $\gamma_{\lambda}$ (solid lines) and its standard deviation (dotted lines) as a function of the distance to the ice edge for the three scenes presented in Fig. 4. It shows that for the cases presented in Fig. $4 \mathrm{~b}$ and $\mathrm{d}$ (red and blue in Fig. 6), close to the detected sea ice areas, narrow bands of enhanced $\gamma_{\lambda}$ are observed, which are most likely related to $3-\mathrm{D}$ radiative effects in clouds and the interaction between clouds and surface. In this study, only the latter case is considered, namely, the 3-D radiative effects related to the pathway of the photons between cloud and surface. Horizon-

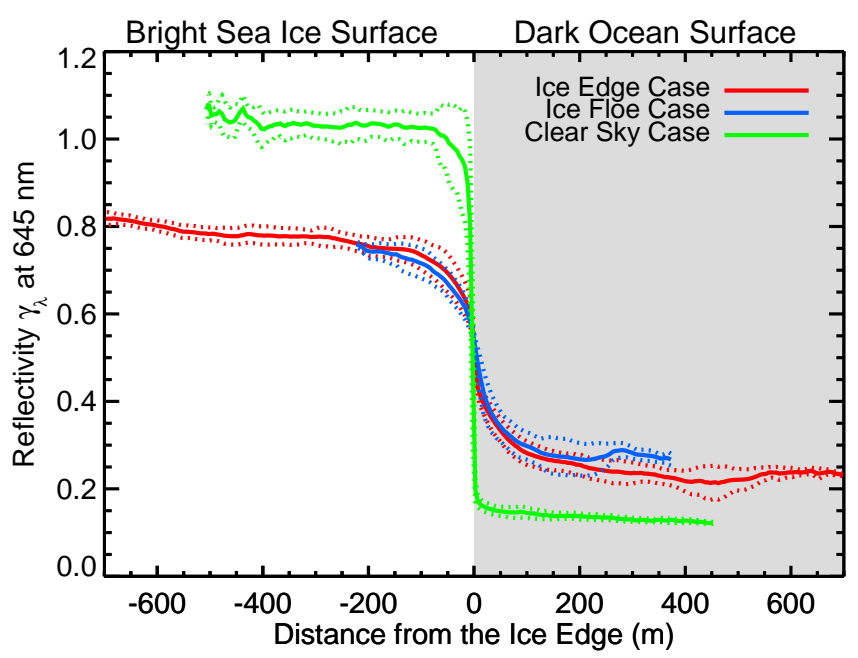

Figure 6. Averaged $\gamma_{\lambda}$ (solid lines) $\pm 6 \%$ measurement uncertainty of $\gamma_{\lambda}$ (dashed lines) at $645 \mathrm{~nm}$ wavelength, measured perpendicular to the ice edges shown in Fig. 4.

tal photon transport in the layer between surface and cloud smoothes the abrupt decrease of the surface albedo from large values above sea ice to low values above the open water. For measurements without clouds (Fig. 4f, green in Fig. 6) we could not find similar areas with enhanced $\gamma_{\lambda}$ above the water close to the ice edge.

The theory explaining the 3-D radiative effect, which causes the enhancement of $\gamma_{\lambda}$, is illustrated in Fig. 7. The incident radiation $\left(F_{0} \cdot \cos (\Theta)\right)$ impinges on the cloud, where scattering and absorption processes take place. Part of the incident radiation is transmitted through the cloud and scattered into the direction of the ice edge (bold black arrow). Sea ice acts similarly to a Lambertian reflector and reflects the incoming radiation almost uniformly in all directions (grey arrows). The reflected radiation penetrates the cloud at a certain altitude (red or blue arrows), from where parts of it are scattered into the observation direction. Without sea ice in the vicinity of the measurements, the reflected radiance would be influenced only by the cloud and dark ocean water. With sea ice in the vicinity, the measured nadir radiance $I_{\lambda}^{\uparrow}$ above the cloud parcel is enhanced due to the additional radiation reflected from the sea ice into the direction of the last scattering point in the cloud. This effect is significant only for cloudy cases, because of the weak scattering efficiency of the clear atmosphere compared to that of clouds. If we compare this effect for clouds of different altitude (Fig. 7), the horizontal photon path of the reflected radiation is extended for clouds of higher altitude (compare for cloud A (red) and cloud B (blue)). Hence, the range of the 3-D effect increases with cloud altitude.

For the case of the straight ice edge (Fig. 4a), the distances presented in Fig. 6 are almost in line with the flight track which was perpendicular to the ice edge. The spatial 


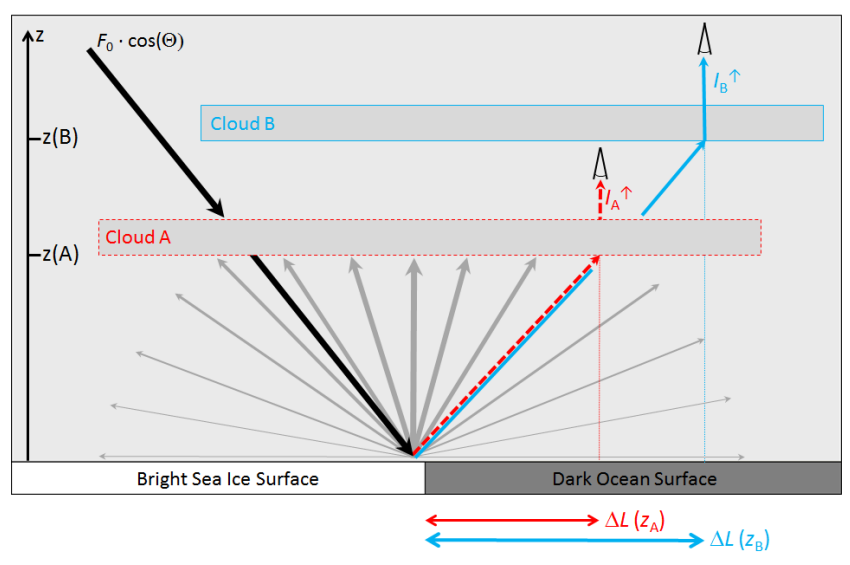

Figure 7. Sketch of the 3-D radiative effects between clouds at two different altitudes and the surface in the vicinity of an ice edge. The arrows illustrate the pathway of the photons between source, cloud, surface, and sensor.

range of $1400 \mathrm{~m}$ perpendicular to the ice edge corresponds to $1418 \mathrm{~m}$ distance along the flight track. With a frame rate of $30 \mathrm{~Hz}$ and an aircraft speed of $65 \mathrm{~m} \mathrm{~s}^{-1}$ this results in 700 measurements along the $1418 \mathrm{~m}$ for each of the 1024 spatial pixels. This large amount of data provides good statistics for the mean $\gamma_{\lambda}$ illustrated as a solid red line in Fig. 6. In general, $\gamma_{\lambda}$ decreases by about two thirds from $\gamma_{\lambda}=0.75$ above bright sea ice to about $\gamma_{\lambda}=0.25$ above dark ocean surface. For scene (a) and (b) the decrease does not occur sharply at the ice edge, but gradually starts at about $400 \mathrm{~m}$ distance from the ice edge and ends at $400 \mathrm{~m}$ distance from the ice edge over open water. In the cloud-free case, the asymptotic values above sea ice and water are reached much closer to the ice edge at about $50 \mathrm{~m}$.

Over the open sea, an enhancement of $\gamma_{\lambda}$ was measured close to the ice edge; while over the ice-covered area, $\gamma_{\lambda}$ is reduced near the ice edge. We define two distances measured from the ice edge to quantify the enhancement effect. The first distance $\Delta L_{\mathrm{HPT}}$ is introduced to quantify the range of horizontal photon transport. It characterizes the distance at which the transition from high $\gamma_{\lambda}$, ice to low $\gamma_{\lambda}$, water is $1 / e^{3}$ of the initial difference between the mean $\gamma_{\lambda}$ above ice $\left(\bar{\gamma}_{\lambda \text {,ice }}\right)$ and the mean $\gamma_{\lambda}$ above open water $\left(\bar{\gamma}_{\lambda \text {,water }}\right)$ :

$\gamma_{\lambda, \text { water }}\left(\Delta L_{\mathrm{HPT}}\right)=\bar{\gamma}_{\lambda, \text { water }}+\frac{1}{e^{3}} \cdot \Delta \mathrm{IPA}$,

with $\Delta \mathrm{IPA}=\bar{\gamma}_{\lambda \text {,ice }}-\bar{\gamma}_{\lambda \text {,water }}$. By including $\Delta \mathrm{IPA}, \Delta L_{\mathrm{HPT}}$ quantifies the range of horizontal photon transport independent of the difference of the surface albedo contrast. For the scene from Fig. 4a (see red line in Fig. 6), $\Delta L_{\mathrm{HPT}}$ indicated by the enhancement of $\gamma_{\lambda}$ over the water surface extends to a distance of $200 \mathrm{~m}$ from the ice edge.

Furthermore, a second distance to the ice edge $\Delta L$ is de-

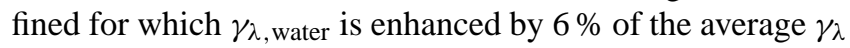

above open water.

$\gamma_{\lambda, \text { water }}(\Delta L)=\bar{\gamma}_{\lambda, \text { water }}+0.06 \cdot \bar{\gamma}_{\lambda, \text { water }}$.

The choice of the threshold results from the radiance measurement uncertainty $( \pm 6 \%)$ of the imaging spectrometer AisaEAGLE. Using this definition, $\Delta L$ is independent of $\gamma_{\lambda}$ measured above the ice surface. It only accounts for the significance of the enhancement with respect to the measurement uncertainty. If the enhancement is higher than the measurement uncertainty, a cloud retrieval might be significantly biased when using the contaminated measurements. Therefore, $\Delta L$ is a measure for the horizontal extent within which the 3-D effects bias the cloud retrieval in the vicinity of an ice edge. For the special case of the measured $\gamma_{\lambda}$ in Fig. 6, the $\Delta L=300 \mathrm{~m}$. Above open water, all measurements within that transition zone cannot be used for the cloud retrieval as the enhanced $\gamma_{\lambda}$ will positively bias the retrieved $\tau$.

For the isolated ice floes of Fig. $4 \mathrm{c}$, the values of the averaged $\gamma_{\lambda}$ (solid blue line) in Fig. 6 are comparable to the values from the scenario in Fig. 4a. Furthermore, Fig. 6 shows a similar analysis (solid green line) for the cloud-free scenario on 14 May 2012 (Fig. 4f). Here, the decrease of $\gamma_{\lambda}$ at the ice edge is significantly sharper than for the cloudcovered scenes. This indicates that in the cloudy scenes horizontal photon transport is taking place in the layer between the bright surface and cloud base, leading to a smoother transition between bright sea ice and dark ocean water.

\subsection{Model studies}

To better quantify the observations of 3-D radiative effects at ice edges, we applied a 3-D radiative transfer model. The simulations are used to determine $\Delta L$ and $\Delta L_{\mathrm{HPT}}$ as a function of different cloud properties. We expect that the interaction between clouds and sea-ice surface varies with varying $\tau$, geometrical thickness, and cloud altitude. Furthermore, the simulations are used to clarify whether these 3-D radiative effects result in an enhancement of the mean $\gamma_{\lambda}$ for a certain area or if the enhancement is, on average, counterbalanced by the decrease of $\gamma_{\lambda}$ above the sea ice. Different idealized sea-ice geometries are studied to investigate the horizontal pattern and average of the 3-D effects.

The radiative transfer simulations of the upwelling radiance $I_{\lambda}^{\uparrow}$ and downwelling irradiance $F_{\lambda}^{\downarrow}$ to derive $\gamma_{\lambda}$ are performed with the open-source Monte Carlo Atmospheric Radiative Transfer Simulator (MCARaTS) which is a forwardpropagating Monte Carlo photon-transport model (Iwabuchi, 2006; Iwabuchi and Kobayashi, 2008). It traces individual photons on their path through the 3-D atmosphere. To reduce the computational effort for radiance simulations, MCARaTS uses several variance-reduction techniques, such as a modified local-estimate method or a truncation approximation for highly anisotropic phase functions (Iwabuchi, 2006). The input to the radiative transfer model contains the optical properties of atmospheric contributions such as 
clouds and trace gases (e.g., extinction coefficients, singlescattering albedos, phase functions) and the 2-D surface albedo. The model requires a predefined albedo field. For this purpose we create a field of $20000 \mathrm{~m}$ by $20000 \mathrm{~m}$ with a pixel size of $50 \mathrm{~m}$ by $50 \mathrm{~m}$ (400 pixels in both horizontal dimensions). Depending on the given sea-ice distribution, the albedo of individual pixels is set to sea-ice albedo $(0.910$ at $645 \mathrm{~nm}$; Bowker et al., 1985) or water albedo (0.042 at $645 \mathrm{~nm}$; Bowker et al., 1985). The pixel size of the 3-D model is about ten times larger than that of AisaEAGLE. However, model results and measurements are still comparable as the investigated 3-D effects occur in the range of a few hundred meters on either side of the ice edge. Accordingly, the AisaEAGLE data are averaged for comparing the model results with the measurements. $2.2 \times 10^{9}$ photons were used in each single model run, which resulted in a noise level of the 3-D simulations of less than $1 \%$. This value is much lower than the measurement uncertainties of AisaEAGLE.

Other input parameters for the model are adapted to the measurement conditions on 17 May 2012 around 17:00 UTC with a solar zenith angle of $58^{\circ}$ and the solar azimuth angle of $113^{\circ}$. The extraterrestrial solar spectrum was taken from Gueymard (2004). The output altitude for $I_{\lambda}^{\uparrow}$ and $F_{\lambda}^{\downarrow}$ is $2920 \mathrm{~m}$ ( $10000 \mathrm{ft}$ flight altitude). To represent the observed clouds in an idealized way, a horizontally and vertically homogeneous liquid water cloud was assumed between 0 and $200 \mathrm{~m}$ altitude. Besides reference simulations for clear-sky conditions, the cloud optical thickness was varied between $\tau=1$ and $\tau=10$ as observed by MODIS in the surroundings of the measurement area. Based on the observations, the effective radius of the liquid water droplets was set to $r_{\text {eff }}=15 \mu \mathrm{m}$. The microphysical properties of the liquid water clouds are converted to optical properties by Mie calculations. Furthermore, profiles of the atmospheric pressure, temperature, density, and gases are taken from profiles given by Anderson et al. (1986). Gas absorption was modelled by LOWTRAN (Low Resolution Transmission Model parametrization, Pierluissi and Peng, 1985), as adapted from SBDART (Santa Barbara DISORT Atmospheric Radiative Transfer, Ricchiazzi and Gautier, 1998).

Additionally, 1-D simulations which use the independent pixel approximation (IPA) are applied to the particular cases. The IPA simulations were performed with the same 3-D model, but with a homogeneous surface albedo - either dark ocean water or bright sea ice. All other parameters remain the same as in the 3-D simulations.

\subsubsection{Infinitely straight ice edge}

The most general case of an ice edge is an infinitely straight ice edge. This case is comparable to Fig. 4a. Figure 8 illustrates the results of the 1-D (grey lines) and 3-D (black lines) simulations. Similar to the observations, $\gamma_{\lambda}$ from the 3-D simulations decreases above the bright sea ice, and increases above the dark ocean surface. The effect is larger the

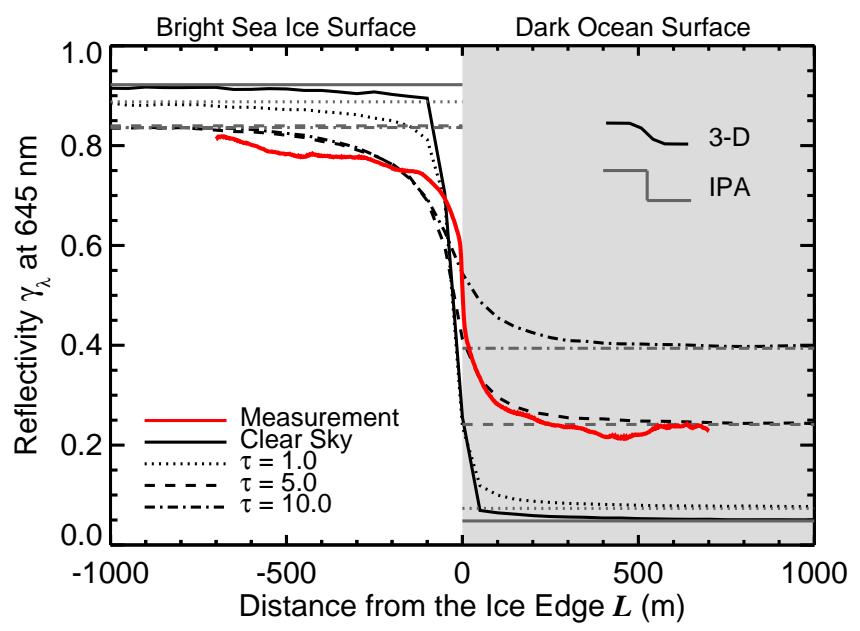

Figure 8. Simulated mean $\gamma_{\lambda}$ across an ice edge for clear-sky conditions as well as for low-level clouds between 0 and $200 \mathrm{~m}$ altitude, $\tau=1 / 5 / 10$, and $r_{\text {eff }}=15 \mu \mathrm{m}$. The white area illustrates the bright sea ice, the grey area and the dark ocean water. Included are the results of the 3-D and IPA simulation, as well as the average of $\gamma_{\lambda}$, measured perpendicular to the ice edge in Fig. $4 \mathrm{a}$.

closer the pixel is located to the ice edge. In the clear-sky simulations this effect is small; 3-D and IPA simulations are almost identical. This indicates that the 3-D effect is dominated by horizontal photon transport between sea ice and clouds and the scattering processes by the cloud particles into the nadir observation direction. Without clouds, the horizontal photon transport above the isotropically reflecting surface is of similar magnitude to the cloudy case. However, due to the weak scattering in the clear atmosphere compared to the scattering by cloud particles, this effect is only significant for cloudy cases. Similar investigations are presented by Marshak et al. (2008) with respect to aerosol-cloud interactions. In the vicinity of clouds, they found that the radiance in cloud-free columns is increased due to a cloud-induced enhancement of the Rayleigh scattering. In general, Fig. 8 shows that with increasing $\tau$ the slope of the decrease of $\gamma_{\lambda}$ next to the ice edge is flattened. This is a result of the reduction in contrast between the dark ocean surface and bright sea ice surface by the overlying clouds.

To compare the results with the measurement example in Fig. 6, the distance $\Delta L_{\mathrm{HPT}}$ defined by Eq. (3) is analyzed. $\bar{\gamma}_{\lambda \text {,water }}$ is set to the IPA values above water. For the cases presented in Fig. 8, $\Delta L_{\mathrm{HPT}}$ increases with increasing $\tau$ from $100 \mathrm{~m}$ at $\tau=1$ to $250 \mathrm{~m}$ at $\tau=5$ and to $300 \mathrm{~m}$ at $\tau=10$. This shows that the horizontal photon transport increases with $\tau$ due to increased scattering inside the cloud layer.

In contrast to $\Delta L_{\mathrm{HPT}}$, the distance $\Delta L$ defined by Eq. (4) decreases from $600 \mathrm{~m}$ (at $\tau=1.0$ ) to $400 \mathrm{~m}$ (at $\tau=5.0$ ) and to $250 \mathrm{~m}$ (at $\tau=10.0$ ). The decrease of $\Delta L$ suggests that the area in which $\gamma_{\lambda}$ is enhanced and a cloud retrieval might be biased is smaller for optically thick clouds. This is re- 


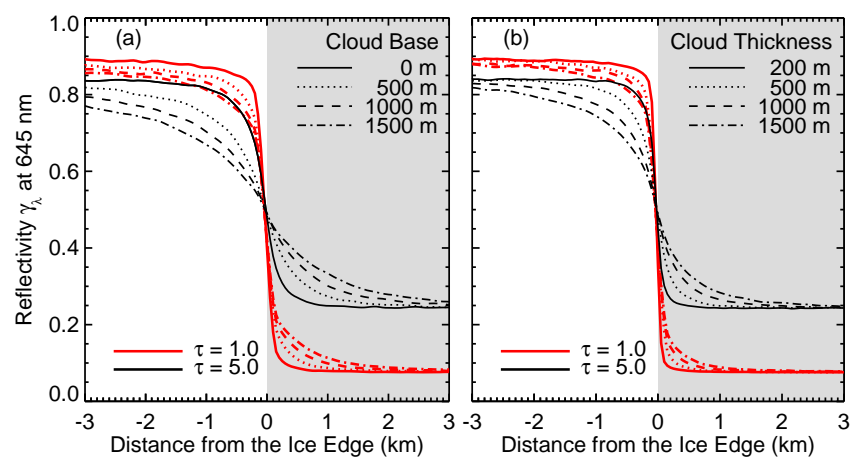

Figure 9. Simulated $\gamma_{\lambda}$ for clouds at different altitudes and with different geometrical thickness for the passage from a highly reflecting ice-covered region to a darker region of open water. The white area illustrates the ice stripe. (a) Simulations for varying cloud base, given a fixed cloud geometrical thickness of $500 \mathrm{~m}$. (b) Simulations for varying cloud geometrical thickness, given a fixed cloud base at $0 \mathrm{~m}$.

lated to the decrease in contrast between cloud covered sea ice and cloud covered ocean if $\tau$ increases. The difference $\Delta$ (IPA) between $\gamma_{\lambda}$,ice and $\gamma_{\lambda}$,water decreases from $\gamma_{\lambda}=0.87$ for the clear-sky case to $\gamma_{\lambda}=0.44$ for $\tau=10$, mainly due to the increasing reflection of incoming radiation by the cloud.

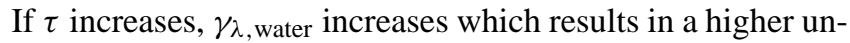
certainty range exceeding the $\gamma_{\lambda}$ enhancement also in areas closer to the ice edge. Therefore, the $\gamma_{\lambda}$ enhancement becomes less significant for a cloud retrieval compared to the measurement uncertainties. Since we aim to retrieve $\tau$ above water areas enclosed by ice floes, in the following $\Delta L$ is used to quantify the 3-D effects.

Additionally, the sensitivity of the results to the assumption of a constant $r_{\text {eff }}$ was analyzed by running simulations with an $r_{\text {eff }}$ of 10,15, 20, and $30 \mu \mathrm{m}$ (not shown here). The results showed that $\Delta L$ is almost independent of $r_{\text {eff }}$. This indicates that a variation of $r_{\text {eff }}$ does not need to be considered when estimating the 3-D radiative effects described here.

Furthermore, simulations with varied values of the surface albedo were performed (not shown). Based on the measurement uncertainty of AisaEAGLE, the surface albedo of the dark ocean water and bright sea ice was varied by $\pm 6 \%$. Over the dark ocean area, the simulations show almost identical results with differences far below $1 \%$ in $\gamma_{\lambda}$. Compared to the measurement uncertainties, those differences in the surface albedo are of less significance for $\Delta L$. Indeed, the albedo has a larger effect over the sea-ice surface (up to $10 \%$ ) due to changing the albedo value relative with $6 \%$, which corresponds to an absolute change of \pm 0.05 compared to 0.002 absolute change for the water surface. For the investigations presented here, the effect over the dark ocean area is relevant only.

Other important aspects which influence the results are the cloud altitude and cloud geometrical thickness. The horizontal photon transport resulting from the horizontal displace-

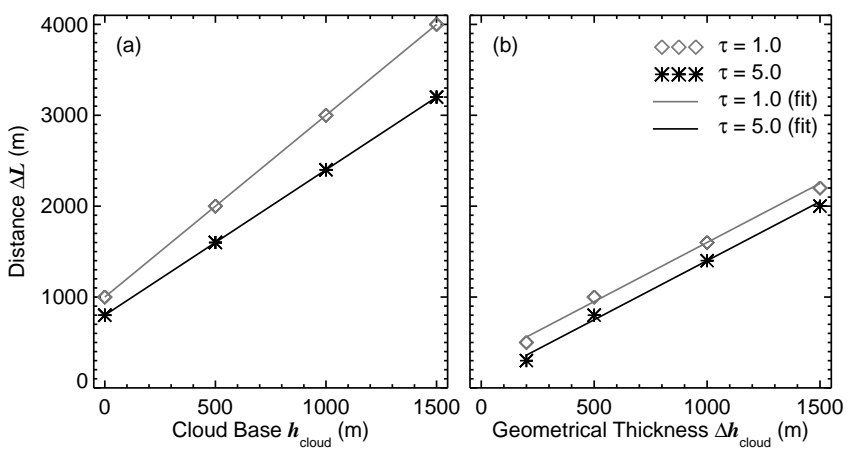

Figure 10. (a) Distance $\Delta L$ as a function of the cloud base altitude $h_{\text {cloud }}$ for a cloud with a geometrical thickness of $\Delta h_{\text {cloud }}=500 \mathrm{~m}$ and different $\tau$. (b) Distance $\Delta L$ as a function of the cloud geometrical thickness $\Delta h_{\text {cloud }}$ for a low-level cloud with cloud base at $h_{\text {cloud }}=0 \mathrm{~m}$ and different $\tau$.

ment of the location where a photon is isotropically scattered at the surface and the location in the cloud where it is scattered afterwards into the viewing direction, changes if the cloud geometry changes. Figure 9 shows the simulated $\gamma_{\lambda}$ for clouds of different altitude (Fig. 9a) and clouds of different geometrical thickness (Fig. 9b). Increasing the cloud base of a cloud with a geometrical thickness of $500 \mathrm{~m}$ and $\tau=1$ from $h_{\text {cloud }}=0 \mathrm{~m}$ to $h_{\text {cloud }}=1500 \mathrm{~m}, \Delta L$ increases from 1000 to $4000 \mathrm{~m}$. For $\tau=5 \Delta L$ increases from 800 to $3200 \mathrm{~m}$. Similarly, $\Delta L$ increases with increasing geometrical thickness. For $\tau=1, \Delta L$ increases from 500 to $2200 \mathrm{~m}$ when the cloud geometrical thickness changes from 200 to $1500 \mathrm{~m}$. For $\tau=5, \Delta L$ increases from 300 to $2000 \mathrm{~m}$. Compared to the influence of $\tau$, the cloud altitude and cloud geometrical thickness have a similar impact on $\Delta L$ and cannot be neglected.

For two model clouds with a geometrical thickness of $500 \mathrm{~m}$ and values of $\tau=1$ and $\tau=5$, Fig. 10a shows $\Delta L$ as a function of the cloud base altitude $h_{\text {cloud }}$. Similarly, Fig. 10b shows $\Delta L$ as a function of the cloud geometrical thickness $\Delta h_{\text {cloud }}$ for low-level clouds with $\tau=1$ and $\tau=5$ and cloud base at $0 \mathrm{~m}$. The increase of $\Delta L$ with increasing altitude of the cloud base (Fig. 10a) follows an almost linear function and can be parameterized by

$\Delta L\left(h_{\text {cloud }}, \tau\right)=A(\tau) \cdot h_{\text {cloud }}+B(\tau)$.

For the parameters $A(\tau)$ and $B(\tau)$, the linear regression yields $A(\tau)=2.00 / 1.6$ and $B(\tau)=1000 \mathrm{~m} / 800 \mathrm{~m}$ for clouds with $\tau=1 / 5$. This shows that the influence on $\Delta L$ is much larger for clouds at higher altitudes and lower $\tau$. Comparing the results for $\tau=1$ and $\tau=5$ indicates that slope $A$ decreases with increasing $\tau$. This proves that the influence of cloud geometry on $\Delta L$ is decreasing with increasing $\tau$.

Similarly, $\Delta L$ increases almost linearly with increasing cloud geometrical thickness $\Delta h_{\text {cloud }}$. This relation can be pa- 
rameterized by

$\Delta L\left(\Delta h_{\text {cloud }}, \tau\right)=A(\tau) \cdot \Delta h_{\text {cloud }}+B(\tau)$.

The regression of the increase of $\Delta L$ with increasing cloud geometrical thickness yield $A(\tau)=1.3 / 1.3$ and $B(\tau)=$ $300 \mathrm{~m} / 100 \mathrm{~m}$ for clouds with $\tau=1 / 5$.

The cloud bases of all boundary layer clouds observed during VERDI ranged between 0 and $650 \mathrm{~m}$ which is in agreement with the climatology presented by Shupe et al. (2011). To demonstrate the potential effects of clouds with higher cloud base, the following simulations cover two cloud cases, one similar to the observed cases from Fig. $4 \mathrm{a}-\mathrm{d}$ ( $h_{\text {cloud }}=0$ $200 \mathrm{~m}$ ) and one with cloud base at $500 \mathrm{~m}$ and cloud top at $1000 \mathrm{~m}$.

\subsubsection{Single circular ice floes}

An infinitely straight ice edge as discussed in Sect. 4.2.1 does not represent reality in all aspects. Scattered ice floes of different size are often observed (see Fig. 4c). In this case, we expect a reduced 3-D effect above open water due to the curvature of the ice edge.

To analyze and quantify this reduction of 3-D radiative effects, we simulated single circular ice floes of different sizes. The radius of the circular ice floe was varied from 100 to $1000 \mathrm{~m}$ in steps of $100 \mathrm{~m}$ and from 1 to $9 \mathrm{~km}$ in steps of $1 \mathrm{~km}$. The center of the circular ice floe was placed in the middle of the model domain. For reasons of symmetry, a cross section through the center of the model domain was used for the data evaluation. Figure 11 shows the influence of the ice-floe size on $\Delta L$.

As expected, $\Delta L$ is lower for small ice floes than for the infinitely straight ice edge simulated in Sect. 4.2.1. For all simulated $\tau, \Delta L$ increases with an increasing radius of the ice floe, asymptotically reaching a maximum value which is identical to the results of the infinitely ice edge $(r=\infty)$. This shows that all ice floes larger than about $6 \mathrm{~km}$ can be treated like the infinitely straight ice edge (for the given cloud and observation geometry).

The reduction of $\Delta L$ for smaller ice floes can be explained by two effects. For ice floes with radii smaller than the distance $\Delta L$ of the infinitely straight ice edge, $r_{\text {floe }}<\Delta L(\tau)$, the size of the ice area is too small for the $\gamma_{\lambda}$ above the ice to reach the IPA $\gamma_{\lambda}$ at any place. All areas of the ice floe are affected by 3-D effects. On the other hand, the area of the ice floe is too small to fully affect the adjacent water area. The water area behind the ice floe limits its effect. For ice floes with a radius larger than $\Delta L$, the IPA $\gamma_{\lambda}$ will be reached at some point on the ice floe. Only the curvature of the floe reduces the 3-D effect above open water. For any water point near the ice edge, the ice area located close to this point is reduced with increasing curvature. The curvature affects both small and large ice floes and lowers the 3-D radiative effects slightly until the maximum effect, which is reached for an infinitely straight ice edge.

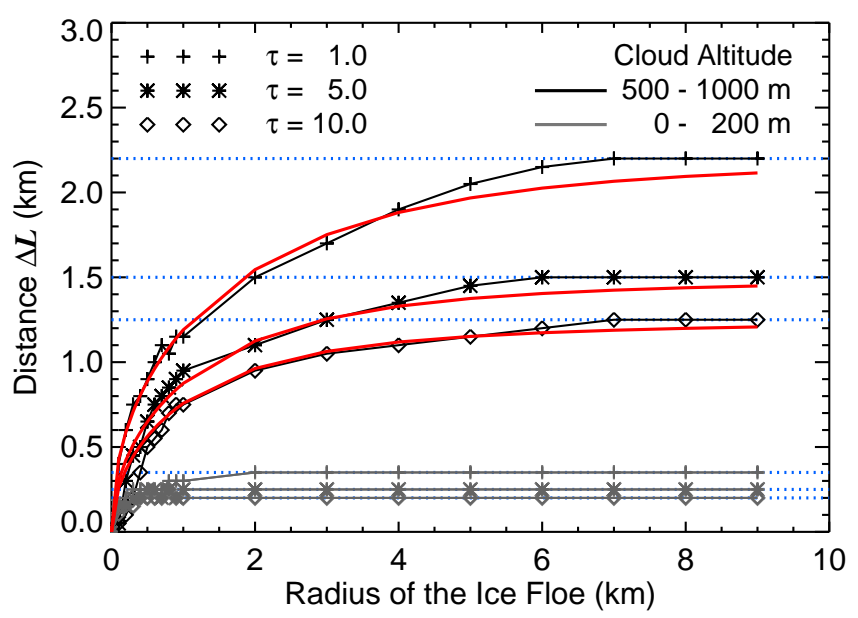

Figure 11. Simulations (grey and black lines) and parametrizations (red lines) of $\Delta L$ as a function of the ice floe size, different $\tau$, and different cloud altitudes. Asymptotic maximum values of $\Delta L$ are marked with dotted blue lines.

Combining these two effects and assuming an exponential relationship between $\Delta L$ and the ice flow size $r_{\text {floe, }}$, we parameterized the results presented in Fig. 11 by

$$
\begin{aligned}
& \Delta L=\frac{2}{3} \cdot \Delta L_{\max }(\tau) \cdot\left[1-\exp \left(-\frac{r_{\text {floe }}^{2}}{C^{2}}\right)\right] \\
& +\frac{1}{3} \cdot \Delta L_{\max }(\tau) \cdot\left[1-\exp \left(-\frac{r_{\text {floe }}}{\Delta L_{\max }(\tau)}\right)\right] .
\end{aligned}
$$

The distances for the infinitely straight ice edge, $\Delta L_{\max }(\tau)$, are taken from Sect. 4.2.1. For the exponential parameter $C$, the fit yields $C=1000 \mathrm{~m}$ for clouds with $\tau=1 / 5 / 10$. The parametrization is valid for ice floes with radii $r_{\text {floe }}$ larger than $300 \mathrm{~m}$. For those ice floes the uncertainty is less than $5 \%$. For ice floes with radii $r_{\text {floe }}$ less than $300 \mathrm{~m}$, the uncertainty increases rapidly and reaches up to $100 \%$. This is due to the insufficient representation of the circular shape of the small ice floes by squared pixels with $50 \mathrm{~m}$ edge length.

\subsubsection{Groups of ice floes}

To get closer to reality, we have to consider ice floes of different sizes creating an irregular mixture of ice and water surfaces. In this case, the size of the ice floes, their shape and the distance to each other influence the described 3-D effects. To address these more complex cases, we ran four simulations with the same model setup as in Sects. 4.2.1 and 4.2.2, but changed the shape and the number of the ice floes. In total, four scenarios with ice floes represented by squares (total sea-ice area $A_{x}$, total ice edge length $l_{x}$ ) were investigated to quantify the influence of $A_{x}$ and $l_{x}$ on the 3-D effects.

In Scenario 1, which serves as a reference, an ice floe with a size of $5 \mathrm{~km}$ by $5 \mathrm{~km}\left(A_{1}=25 \mathrm{~km}^{2}, l_{1}=20 \mathrm{~km}\right)$ was placed 


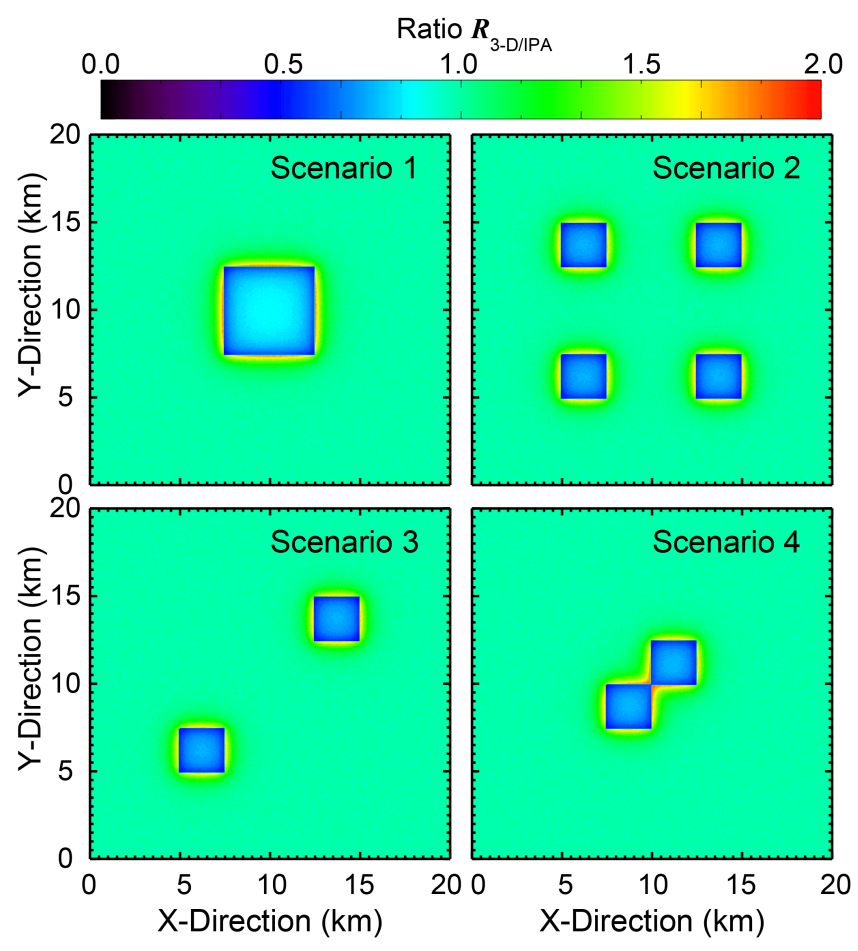

Figure 12. Ratio $R_{3-\mathrm{D} / \mathrm{IPA}}$ of $\gamma_{\lambda}$ of the 3 -D and IPA simulation at $\tau=5$ and for a cloud between 500 and $1000 \mathrm{~m}$ altitude. Each panel displays one of the four scenarios.

in the center of the model domain. For Scenario 2, the total sea-ice area was conserved $\left(A_{2}=A_{1}=25 \mathrm{~km}^{2}\right)$ and the total ice edge length doubled $\left(l_{2}=2 \cdot l_{1}=40 \mathrm{~km}\right)$. This was realized by four smaller ice floes $(2.5 \mathrm{~km}$ by $2.5 \mathrm{~km})$, separated by a distance of $5 \mathrm{~km}$ from each other. Scenario 3 simulates two small ice floes $(2.5 \mathrm{~km}$ by $2.5 \mathrm{~km})$ for which the total ice edge length has been conserved $\left(A_{3}=0.5 \cdot A_{1}=12.5 \mathrm{~km}^{2}\right.$, $\left.l_{3}=l_{1}=20 \mathrm{~km}\right)$. Scenario 4 was designed to investigate the effect of the distance between the single ice floes. The two ice floes of Scenario $3\left(A_{4}=0.5 \cdot A_{1}=12.5 \mathrm{~km}^{2}, l_{4}=l_{1}=\right.$ $20 \mathrm{~km})$ were copied, but placed next to each other.

To highlight the 3-D radiative effects, the ratios $R_{3-\mathrm{D} / \mathrm{IPA}}$ of the 3-D and IPA results are calculated. If both simulations are equal, the ratio is $R_{3-\mathrm{D} / \mathrm{IPA}}=1$. Compared to the IPA simulations, a ratio of $R_{3-\mathrm{D} / \mathrm{IPA}}>1$ represents an enhancement of $\gamma_{\lambda}$. Table 1 lists $R_{3 \text {-D/IPA }}$ for all simulated $\tau$ and $h_{\text {cloud }}$. Figure 12 displays the $R_{3 \text {-D/IPA }}$ for a cloud with $\tau=5$ at $h_{\text {cloud }}=500-1000 \mathrm{~m}$, since the 3-D effects are more evident for larger $\tau$ and higher cloud altitudes. Yet, the overall 3-D effect is relatively small with $R_{3-\mathrm{D} / \mathrm{IPA}}$ ranging from 96.5 to $98.4 \%$, but is still significantly above the noise level of the 3-D simulations. The small values are caused by the large model domain and the relatively small ice fraction. Most pixels are ice-free and at a distance to the ice floes where 3-D effects are negligible.

As expected, in each scenario the contrast at the ice floe boundaries is reduced by the 3-D effects. To quantify the 3-D

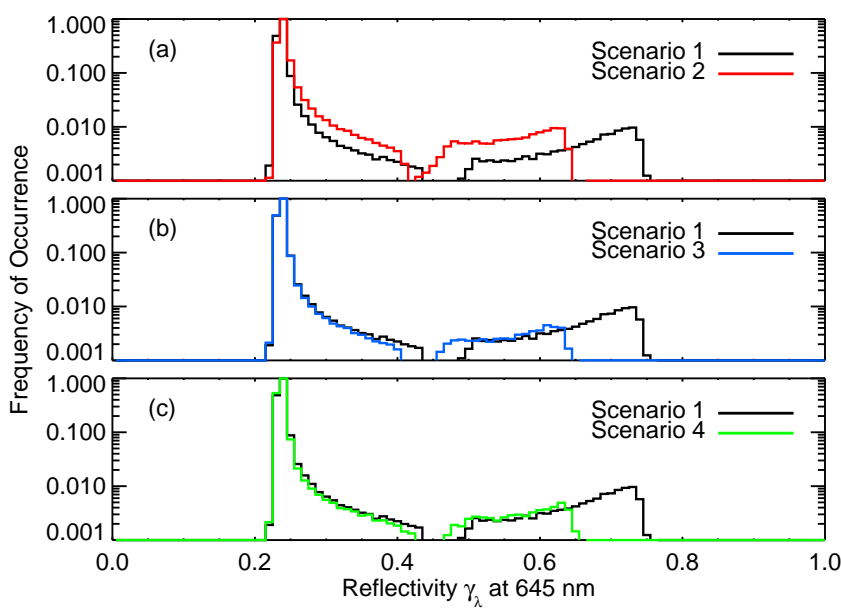

Figure 13. Single logarithmic frequency distributions of the modelled $\gamma_{\lambda}$ from all four scenarios displayed in Fig. 12.

radiative effect in the entire model domain, average and standard deviations (SD) of $\gamma_{\lambda}$ are calculated (not listed). The average $\gamma_{\lambda}$ is largest for Scenario 1 and smallest for Scenarios 3 and 4 . This is not surprising since the area of the ice floes in Scenario 3 and 4 is half the area of the reference scenario. In all scenarios, evidence of the 3-D radiative effect is found in a slight reduction of the average $\gamma_{\lambda}\left(R_{\text {total }}<1\right)$. This reduction originates from the absorption of the radiation, which is scattered by the cloud base back into the direction of the dark ocean surface. This part of absorption does not exist in the IPA simulations, which in comparison leads to a lower scene average reflection in the 3-D simulations. The SDs in the 3-D simulations are also lower than in the IPA results, which corresponds to the smoothing at the ice edges.

In Scenarios 1-3, the individual ice floes do not affect each other, as the distance between them is too large. Here, similar 3-D effects are observed. The corners of the ice floes are smoothed out and the $\gamma_{\lambda}$ field around the ice floe becomes more circular. This results in larger $\Delta L$ values at the center of the ice edges and in smaller $\Delta L$ values close to the corners. For the large ice floe of Scenario $1, \Delta L=1400 \mathrm{~m}$ at the center of each side. In agreement with Fig. 11, $\Delta L$ for the small ice floes in Scenario 2 and 3 is smaller $(1200 \mathrm{~m})$. Along the normal line of the corners, $\Delta L$ is reduced to $900 \mathrm{~m}$ for Scenario 1 and $700 \mathrm{~m}$ for Scenario 2 and 3. In Scenario 4 , the ice floes cause a different pattern close to their point of contact. Here, $\Delta L$ is largest measured tangential to the connected corners and reaches $2000 \mathrm{~m}$ as the 3 -D radiative effects of both floes add up for these points.

In addition, Fig. 13 shows frequency distributions of $\gamma_{\lambda}$ for the four scenarios. Since the clouds in the simulations are homogeneous, the $\gamma_{\lambda}$ in areas unaffected by 3 -D effects are identical to the $\gamma_{\lambda}$ from the IPA simulation. For the openwater pixels, this causes a single peak at $\gamma_{\lambda}(\tau=5)=0.24$. For pixels above ice, no single peak corresponding to the 
Table 1. Ratio $R_{3-\mathrm{D} / \mathrm{IPA}}$ (in \%) of $\gamma_{\lambda}$ for the total scene area $\left(R_{\text {total }}\right)$, for the sea-ice covered area $\left(R_{\text {ice }}\right)$, and for the dark ocean covered area ( $R_{\text {water }}$ ) of all scenarios from Sects. 4.2.3 and 4.2.4. The simulations for Scenario $1-4$ are performed with clouds of $\tau=1$ and $\tau=5$ at an altitude of 0-200 and 500-1000 m. The simulation for the real case is performed with a cloud of $\tau=5$ at an altitude of 100-200 $\mathrm{m}$.

\begin{tabular}{|c|c|c|c|c|c|c|c|}
\hline $0-200 \mathrm{~m}$ & Case & $\begin{array}{r}\tau=1 \\
R_{\text {total }}(\%)\end{array}$ & $R_{\text {ice }}(\%)$ & $R_{\text {water }}(\%)$ & $\begin{array}{r}\tau=5 \\
R_{\text {total }}(\%)\end{array}$ & $R_{\text {ice }}(\%)$ & $R_{\text {water }}(\%)$ \\
\hline & 1 & 99.7 & 96.1 & 103.0 & 99.5 & 95.1 & 100.7 \\
\hline & 2 & 99.1 & 94.1 & 104.3 & 99.1 & 91.7 & 101.6 \\
\hline & 3 & 100.0 & 94.0 & 103.0 & 99.5 & 91.7 & 100.7 \\
\hline & 4 & 100.1 & 94.2 & 104.8 & 99.5 & 91.8 & 101.2 \\
\hline & Real Case & - & - & - & 92.6 & 73.2 & 124.0 \\
\hline \multirow[t]{5}{*}{$500-1000 \mathrm{~m}$} & Case & $\begin{array}{r}\tau=1 \\
R_{\text {total }}(\%)\end{array}$ & $R_{\text {ice }}(\%)$ & $R_{\text {water }}(\%)$ & $\begin{array}{r}\tau=5 \\
R_{\text {total }}(\%)\end{array}$ & $R_{\text {ice }}(\%)$ & $R_{\text {water }}(\%)$ \\
\hline & 1 & 97.4 & 87.5 & 107.2 & 97.4 & 77.5 & 103.2 \\
\hline & 2 & 96.5 & 82.5 & 112.8 & 96.5 & 66.5 & 106.0 \\
\hline & 3 & 98.4 & 82.3 & 106.7 & 97.9 & 66.5 & 102.8 \\
\hline & 4 & 98.4 & 82.9 & 109.4 & 98.1 & 67.5 & 103.9 \\
\hline
\end{tabular}

IPA $\gamma_{\lambda}(\tau=5)=0.84$ is observed. This is due to the small size of the ice floes, above which the IPA $\gamma_{\lambda}$ is not reached. All $\gamma_{\lambda}$ values that are not included in the single water peak result from the 3-D effects. Above ice, the distributions of Scenarios 2-4 are shifted to lower $\gamma_{\lambda}$ compared to Scenario 1 . This is because the diameter of the floes is even smaller than in Scenario 1. Thus, the large reflectivity values of Scenario 1 cannot be reached by Scenarios 2-4 (compare Fig. 11). Comparing the frequency distribution of Scenarios 1 and 2, Fig. 13a reveals the effect of the increased ice edge length in Scenario 2. More pixels are affected by 3$\mathrm{D}$ effects and show values different from IPA. Furthermore, with four times the number of corners in Scenario 2, $\gamma_{\lambda}$ above water is slightly shifted to lower values compared to the reference case. Similar effects can be observed in Scenario 3. In Scenario 4, the combined effect of both floes leads to larger enhancements above ocean water than in Scenarios 2 and 3. In comparison to Scenario 3, the reduced distance between the ice floes in Scenario 4 leads to a shift of the frequency distribution over water to larger $\gamma_{\lambda}$ values. Similar results can be observed for the frequency distribution over ice.

In order to identify the relevant effects, we separately calculated the ratios $R_{3-\mathrm{D} / \mathrm{IPA}}$ for ice-free $\left(R_{\text {water }}\right)$ and icecovered $\left(R_{\text {ice }}\right)$ areas (Table 1$)$. The reduction of $\gamma_{\lambda}$ above ice is much larger $\left(R_{\text {ice }}=82-88 \%\right)$ than the enhancement above water $\left(R_{\text {water }}=107-113 \%\right)$, which again is partly a result of the larger water area. But also the small size of the ice floes leads to the stronger effects above sea ice, as the IPA $\gamma_{\lambda}$ is never reached. Interestingly, with increasing $\tau$, the deviations from IPA increase for the ice area but decrease for the dark ocean area. This effect is related to the asymmetry of enhancement and reduction for clouds of high $\tau$, as shown in Fig. 8.

The average reduction over sea-ice areas $\left(R_{\text {ice }}\right)$ is largest for Scenario 1. For all other scenarios, smaller values are ob- tained, with Scenarios 2 and 3 showing values identical to each other. This indicates that the particular distance between the ice floes in Scenarios 2 and 3 is large enough to suppress any influence of the single ice floes on each other. Contrarily, Scenario 4 gives the second largest $R_{\text {ice. }}$. Compared to Scenario 1 and Scenario 3, this confirms that the distance between the ice floes can have a significant influence on the enhancement of $\gamma_{\lambda}$ over dark ocean water in the vicinity of ice edges.

$R_{\text {water }}$ is almost equal for Scenarios 1 and 3, although the ice-covered area differs by a factor of two. For Scenario 2, with a doubled ice boundary length, this ratio is significantly larger. This leads to the conclusion that the ice boundary length has a significantly larger effect on the enhancement of $\gamma_{\lambda}$ (over the water next to an ice edge) than the size of the area covered by sea ice.

\subsubsection{Realistic sea-ice scenario}

In order to combine all aspects of the 3-D effects demonstrated before, $\gamma_{\lambda}$ was simulated above an albedo field generated from the observation shown in Fig. 4c. To simulate ice floes in the same size range as for the measurements, the pixel size of the albedo map was adjusted to the pixel number and size of the AisaEAGLE measurements ( 488 by 601 pixels with $5 \mathrm{~m}$ edge length).

The albedo map was used in the simulations implementing a cloud of $\tau=5$ and a fixed $r_{\mathrm{eff}}=15 \mu \mathrm{m}$, as derived from in situ measurements. With regard to the AMALi measurements, the cloud top altitude was set to $h_{\text {cloud, top }}=200 \mathrm{~m}$. Compared to the simulations shown before, the best agreement between measurement and simulation is derived for this specific case for a cloud base altitude of $h_{\text {cloud, base }}=100 \mathrm{~m}$ and a slightly adjusted surface albedo $\left(\alpha_{\text {water }}=0.09, \alpha_{\text {ice }}=\right.$ 0.83 ). Figure 14 shows the frequency distributions of simu- 
lated and observed $\gamma_{\lambda}$. Comparing observation and simulation, the maximum of the ocean-water and sea-ice peak are found at equal $\gamma_{\lambda}$. In regions over dark ocean water as well as in regions over bright sea ice, the $\gamma_{\lambda}$ of the observation show a broader distribution than the $\gamma_{\lambda}$ of the simulation. Indeed, the magnitude of the simulated $\gamma_{\lambda}$ peak above the seaice surface agrees well with the peak from the observation, while the difference above the dark ocean water is significantly larger. The different magnitude and the broader distribution of the observed single peaks compared to the simulation result most likely from simplifications in the simulations where a horizontally homogeneous cloud is assumed. Thus, variations of $\gamma_{\lambda}$ due to cloud 3-D effects are not included here. Only the surface 3-D effects cause a broadening of the frequency distribution. However, while surface effects will fill up the gap between the two peaks only, cloud inhomogeneities can also result in values smaller (over water) and higher (over sea ice) than the IPA simulations.

In addition to the results from the idealized scenarios in Sect. 4.2.3, Table 1 shows the ratios $R_{3 \text {-D/IPA }}$ between the results of the 3-D and IPA simulation for the realistic sea-ice scenario. Compared to the idealized scenarios in Sect. 4.2.3, for the realistic sea-ice scenario the differences between the IPA and 3-D simulations are larger above dark ocean water and smaller above bright sea ice. This behavior is related to the larger sea-ice fraction in the realistic sea-ice distribution, where water pixels are surrounded by more ice area compared to the isolated sea-ice floes of Sect. 4.2.3. On the one hand, it shows that the main characteristics of the ice-edge induced 3-D radiative effects in clouds can be studied by using idealized surface albedo fields. On the other hand, in case of a real sea-ice distribution from measurements, it is also necessary to consider the real surface-albedo distribution for deriving $\Delta L$ and the overestimation in the retrieved

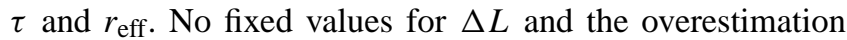
can be given as a function of $\tau$ and cloud altitude alone. The surface-albedo distribution plays a major role as well and has to be known.

\section{Retrieval of cloud optical thickness $\tau$ and effective radius $r_{\text {eff }}$}

All simulations in Sect. 4.2 showed that $\gamma_{\lambda}$ over open water areas close to sea ice can be enhanced drastically if clouds are present. For a cloud retrieval following the strategy by Bierwirth et al. (2013), this enhancement suggests that $\tau$ will be overestimated in this area when a surface albedo of water is assumed. To quantify the magnitude of this overestimation, a synthetic cloud retrieval is investigated. The retrieval is based on simulations only in order to investigate also the uncertainties of retrieved $r_{\text {eff }}$, which cannot be derived from the current setup of AisaEAGLE measurements during VERDI. The limitation of AisaEAGLE to visible wavelengths restricts the retrieval to $\tau$ (Bierwirth et al.,

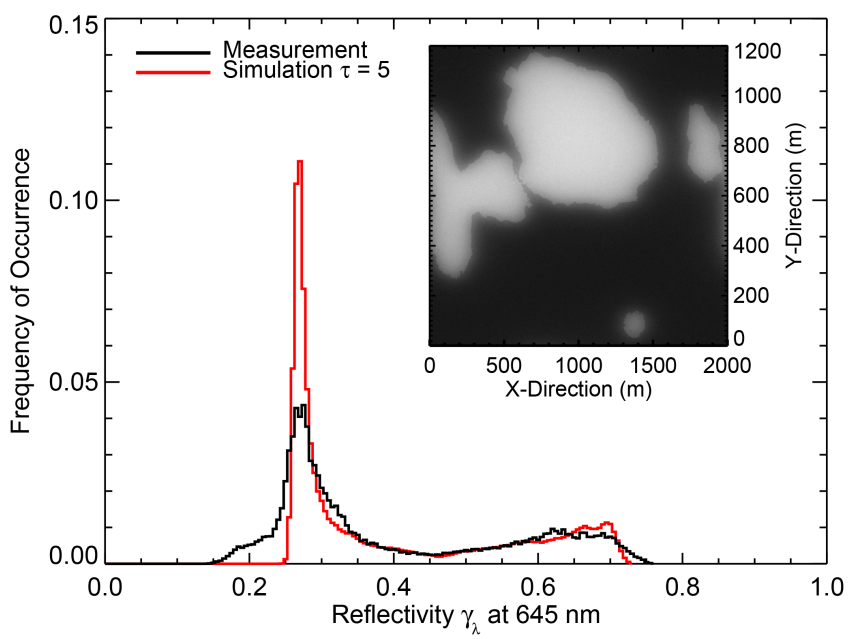

Figure 14. Frequency distributions of measurement and simulation. 3-D simulation performed for the second measurement case, presented in Fig. 4 and Sect. 3. The bin size in $\gamma_{\lambda}$ is 0.005 .

2013). However, near-infrared measurements might be available by use of additional imaging spectrometers such as the AisaHAWK. Therefore, this study addresses both quantities $\tau$ and $r_{\text {eff. }}$. To do so, the retrieval based on the best fit to the forward simulations is applied to the $\gamma_{\lambda}$ field of a 3-D simulation where the cloud optical properties are predetermined. To study a simple case, in the 3-D simulation an isolated ice floe with a radius of $6 \mathrm{~km}$ (Sect. 4.2.2) was chosen and a homogeneous cloud with $\tau=10$ and $r_{\text {eff }}=15 \mu \mathrm{m}$ was placed above it at an altitude of 500 to $1000 \mathrm{~m}$. With a radius of $6 \mathrm{~km}$ the ice floe has an effect similar to that of an infinitely straight ice edge which leads to the maximum range of 3-D effects with the largest $\Delta L$ (see Fig. 11). The retrieval is only performed over the dark ocean surface. The forward simulations of the $\gamma_{\lambda}$ look-up table are based on 1-D simulations. $\tau$ and $r_{\text {eff }}$ are varied in the range of $1-25$ and $10-25 \mu \mathrm{m}$, respectively; see Fig. 15. The retrieval grid is constructed from the simulated $\gamma_{\lambda}$ at $645 \mathrm{~nm}$ wavelength on the abscissa and the ratio of $\gamma_{\lambda}$ at 1525 and $579 \mathrm{~nm}$ wavelength on the ordinate. This wavelength and the wavelength ratio was chosen in order to improve the retrieval method by Bierwirth et al. (2013). The choice of wavelength follows the method presented by Werner et al. (2013). This method creates a retrieval grid with a more separated solution space for $\tau$ and $r_{\text {eff }}$ than the classic two-wavelength method by Nakajima and King (1990) or Bierwirth et al. (2013). Furthermore, it effectively corrects the retrieval results for the influence of overlying cirrus and reduces the retrieval error for $\tau$ and $r_{\text {eff }}$ caused by calibration uncertainties (Werner et al., 2013). For airborne investigations of $\tau$ and $r_{\text {eff }}$ with large spatial coverage and high spatial resolution, this will result in a higher accuracy of the retrieved cloud properties.

The $\gamma_{\lambda}$ of the 3-D simulation is plotted in Fig. 15 as dots colour-coded with the distance to the ice edge. The exact re- 


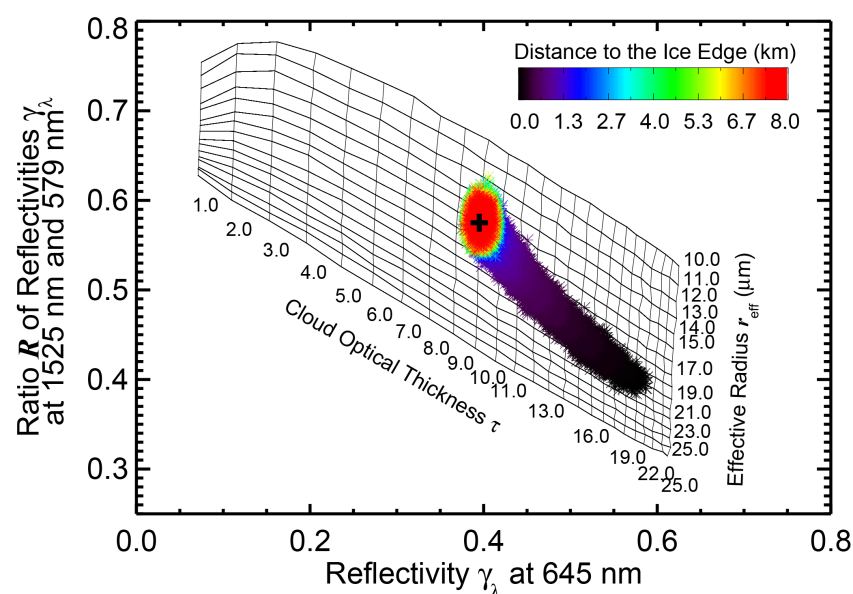

Figure 15. Retrieval grid using $\gamma_{\lambda}$ at $645 \mathrm{~nm}$ and the ratio of $\gamma_{\lambda}$ at $\lambda_{1} / \lambda_{2}=1525 \mathrm{~nm} / 579 \mathrm{~nm} . \gamma_{\lambda}$ of the 3 -D simulation are illustrated by colour-coded dots as a function of distance to the ice-floe edge. The black cross marks the exact cloud properties $\tau=10$ and $r_{\text {eff }}=$ $15 \mu \mathrm{m}$ used in the 3-D simulation for the cloud at 500 to $1000 \mathrm{~m}$ altitude.

sult of a cloud with $\tau=10$ and $r_{\text {eff }}=15 \mu \mathrm{m}$ is marked with a black cross. The results imply a significant overestimation of $\tau$ and $r_{\text {eff }}$ at distances below $2 \mathrm{~km}$ from the ice edge (dark blue dots). The overestimation increases with decreasing distance to the ice edge. As expected, for distances larger than $2 \mathrm{~km}$ from the ice edge (light blue to red dots) the $\gamma_{\lambda}$ is close to the IPA value (black cross). Small deviations are results of noise in the 3-D simulations. For the range below $\Delta L=2 \mathrm{~km}$, the mean $\tau$ and $r_{\text {eff }}$ (solid lines) and their standard deviation (dotted lines) derived from the retrieval are shown as a function of the distance to the ice edge in Fig. 16.

The graph shows that the overestimation of $\tau$ increases up to $90 \%$ while $r_{\text {eff }}$ is biased by up to $30 \%$ close to the ice edge. Both values are valid only for the cloud used in the simulations $\left(\tau=10\right.$ and $\left.r_{\text {eff }}=15 \mu \mathrm{m}\right)$. For a smaller $\tau$, the effect will be reduced. Furthermore, Fig. 16 shows that the overestimation of $\tau$ increases approximately exponentially starting at about $1.5 \mathrm{~km}$ distance, while the overestimation of $r_{\text {eff }}$ increases more slowly and only extends up to a distance of $1.0 \mathrm{~km}$. This indicates that the magnitude of the 3-D effects depends on the wavelengths. In all simulations shown in Sect. 4.2, a wavelength of $645 \mathrm{~nm}$ was used for the retrieval of $\tau$. However, the retrieval of $r_{\text {eff }}$ also requires simulations at $1525 \mathrm{~nm}$ in the absorption band of liquid water. Therefore, the smaller magnitude and horizontal extent of the overestimation of $r_{\text {eff }}$ compared to the magnitude and horizontal extent of the overestimation of $\tau$ suggest that the 3-D effects will be smaller at absorbing wavelengths.

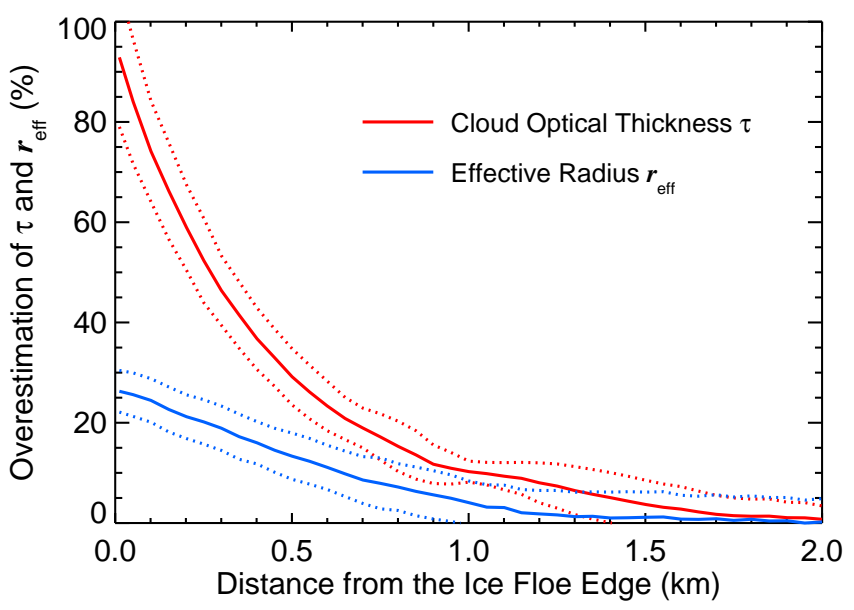

Figure 16. Overestimation (average and standard deviation) of $\tau$ and $r_{\text {eff }}$ as a function of the distance to the edge of the ice floe. The model cloud at an altitude of 500 to $1000 \mathrm{~m}$ had $\tau=10$ and $r_{\text {eff }}=15 \mu \mathrm{m}$.

\section{Summary and conclusions}

During the international field campaign VERDI, airborne measurements of $\gamma_{\lambda}$ were performed with the imaging spectrometer AisaEAGLE and the SMART-Albedometer spectrometer system. In particular, measurements above clouds in situations with heterogeneous surface albedo were analyzed in order to retrieve $\tau$. Due to the high contrast in the surface albedo of sea ice and open water, the data showed a distinct difference between $\gamma_{\lambda}$ above water and sea ice surfaces. This transition was used to distinguish between these surface types. Threshold $\gamma_{\lambda}$ values derived from both measurements and radiative transfer simulations are in good agreement and were found to be robust for the separation of the surfaces.

In the vicinity of ice edges, we found that $\gamma_{\lambda}$ is reduced and/or enhanced above the bright sea ice/dark ocean surface. This is related to 3-D effects, which result from isotropic reflection on the bright sea ice. This reflection causes horizontal photon transport, before the radiation is scattered by cloud particles into the direction of observation. To quantify the uncertainties of a cloud retrieval in such areas of open water close to ice floes, $\gamma_{\lambda}$ measurements from the VERDI campaign were analyzed and 3-D radiative transfer simulations were performed with MCARaTS for a clear-sky case and clouds of $\tau=1 / 5 / 10$ located above various, idealized surface albedo fields. Two distances $\Delta L_{\mathrm{HPT}}$ and $\Delta L$ were defined to characterize the extent of the horizontal photon transport $\left(\Delta L_{\mathrm{HPT}}\right)$ and to estimate the distance to the ice edge $(\Delta L)$ within which the retrieval of $\tau$ and $r_{\text {eff }}$ is biased by the 3 -D effects stronger than by measurement uncertainties.

From the two measurement cases presented here $(\tau=$ $5, h_{\text {cloud }}=0-200 \mathrm{~m}$ ), a distance $\Delta L$ of $400 \mathrm{~m}$ was observed. Radiative transfer simulations, adapted to the ob- 
served cloud and sea-ice situation, confirmed this value. For the case of the infinitely straight ice edge, a distance $\Delta L_{\mathrm{HPT}}=100 \mathrm{~m} / 250 \mathrm{~m} / 300 \mathrm{~m}$ was found for $\tau=1 / 5 / 10$. The increase of $\Delta L_{\mathrm{HPT}}$ shows that the horizontal photon transport is increasing with increasing $\tau$. However, the minimum distance $\Delta L$ to the ice edge, where a 1-D cloud retrieval can be applied is decreasing with increasing $\tau(\Delta L=$ $600 \mathrm{~m} / 400 \mathrm{~m} / 250 \mathrm{~m}$ at $\tau=1 / 5 / 10$ ) due to the stronger impact of measurement uncertainties in case of thicker clouds (higher $\gamma_{\lambda}$ ).

Furthermore, it was found that variations in $r_{\text {eff }}$ do not have to be considered. The simulations did not show significant differences of $\Delta L_{\mathrm{HPT}}$ and $\Delta L$ assuming various $r_{\mathrm{eff}}$ between 10-30 $\mu \mathrm{m}$. Besides cloud properties, the influence of the magnitude of the albedo contrast was tested. Varying the simulated surface albedo of the bright sea ice and dark ocean water by $\pm 6 \%, \Delta L_{\mathrm{HPT}}$ and $\Delta L$ were found to vary less by $<1 \%$ which is less than the given measurement uncertainty of $6 \%$. This indicates that $\Delta L_{\mathrm{HPT}}$ and $\Delta L$ are robust measures that quantify the horizontal extent of the 3-D radiative effect at various albedo contrasts and can be applied as well for e.g. albedo contrast in regions with heterogeneous distributions of forest and deforested areas.

The cloud altitude and cloud geometrical thickness were found to be parameters significantly influencing $\Delta L$. The distance $\Delta L$ increases linearly with an increasing cloud base altitude (for $\tau=5$ from 800 to $3200 \mathrm{~m}$ for a $500 \mathrm{~m}$ thick cloud with cloud base at 0 and $1500 \mathrm{~m}$ ). The same increase of $\Delta L$ holds for an increasing cloud geometrical thickness (for $\tau=5$ from 300 to $2000 \mathrm{~m}$ for a 200 and $1500 \mathrm{~m}$ thick cloud). Therefore, the cloud base altitude and cloud geometrical thickness have to be known exactly while performing 3-D radiative transfer simulations of clouds above ice edges.

The size of the individual ice floe has an influence on $\Delta L$, which increases with an increasing radius of the ice floe, until it asymptotically reaches a maximum value $(\Delta L=2200 \mathrm{~m} / 1500 \mathrm{~m} / 1250 \mathrm{~m}$ for $\tau=1 / 5 / 10$, $h_{\text {cloud }}=500-1000 \mathrm{~m}$, and $r_{\text {floe }} \geq 6 \mathrm{~km}$ ).

To investigate any changes in the 3-D radiative effect due to changes in the ice-edge boundary length or sea-ice area, area-averaged $\gamma_{\lambda}$ were calculated for different idealized cases of sea-ice distributions. A larger enhancement of the areaaveraged $\gamma_{\lambda}$ was found for longer ice edge lengths. Changes in the sea-ice area are of less importance. Placing the ice floes directly next to each other, an enhancement of the areaaveraged $\gamma_{\lambda}$ was found as well, although the sea-ice area and ice-edge length remained the same.

For the direct comparison of simulation and measurement, a realistic ice floe field was modeled. The frequency distributions of observations and simulations agree within the measurement uncertainties. However, the area-averaged $\gamma_{\lambda}$ showed stronger 3-D effects for the real case compared to the idealized cases simulated before. This indicates that an exact quantification of the appearing 3-D radiative effects in clouds above ice edges can only be derived by simulations if realistic surface albedo fields are applied. However, a parameterization of the influence of individual parameters is only possible by using such simplified surface albedo fields.

The results from the simulations suggest that applying a 1D cloud retrieval to airborne measurements taken over ocean areas located close to sea ice edges will result in an overestimation of $\tau$ and $r_{\text {eff }}$ and the overestimation will increase with proximity to the ice edge. This overestimation was calculated for a liquid water cloud with $\tau=10.0$ and $r_{\text {eff }}=15 \mu \mathrm{m}$. In that case, the overestimation of the retrieved $\tau$ reaches up to a distance of $1.5 \mathrm{~km}$ from the ice edge, with a maximum overestimation of $90 \%$ directly beside the ice edge. For $r_{\text {eff }}$, an overestimation of $30 \%$ was found in the direct vicinity of the ice edge. 3-D influences on the retrieval of $r_{\text {eff }}$ are observable up to a distance of $1.0 \mathrm{~km}$ from the ice edge. This is slightly lower compared to the distance where $\tau$ is biased, which indicates that the 3-D effect probably depends on wavelength. Further investigations and the application of similar studies to satellite observations and retrievals of cloud properties with respect to 3-D radiative effects of the heterogeneous surface albedo in Arctic regions will be part of future studies.

Acknowledgements. We are grateful to the Alfred Wegener Institute Helmholtz Centre for Polar and Marine Research, Bremerhaven, Germany for supporting the VERDI campaign with the aircraft and manpower. In addition we like to thank Kenn Borek Air Ltd., Calgary, Canada for the great pilots who made the complicated measurements possible. For excellent ground support with offices and accommodations during the campaign we are grateful of the Aurora Research Institute, Inuvik, Canada.

Edited by: M. Shupe

\section{References}

Anderson, G., Clough, S., Kneizys, F., Chetwynd, J., and Shettle, E.: AFGL Atmospheric Constituent Profiles $(0-120 \mathrm{~km})$, Tech. Rep. AFGL-TR-86-0110, AFGL (OPI), Hanscom AFB MA 01736, 1986.

Armstrong, R. L. and Brodzik, M. J.: Recent Northern Hemisphere Snow Extent: A Comparison of Data Derived from Visible and Microwave Satellite Sensors, Geophy. Res. Lett., 28, 3673-3676, 2001.

Bierwirth, E., Ehrlich, A., Wendisch, M., Gayet, J.-F., Gourbeyre, C., Dupuy, R., Herber, A., Neuber, R., and Lampert, A.: Optical thickness and effective radius of Arctic boundary-layer clouds retrieved from airborne nadir and imaging spectrometry, Atmos. Meas. Tech., 6, 1189-1200, doi:10.5194/amt-6-1189-2013, 2013.

Bowker, D., Davis, R., Myrick, D., Stacy, K., and Jones, W.: Spectral Reflectances of Natural Targets for Use in Remote Sensing Studies, NASA RP-1139, NASA Langley Research Center, Hampton (VA), USA, 1985.

Coddington, O. M., Pilewskie, P., and Vukicevic, T.: The Shannon information content of hyperspectral shortwave cloud albedo 
measurements: Quantification and practical applications, J. Geophy. Res., 117, D04205, doi:10.1029/2011JD016771, 2012.

Grosvenor, D. P. and Wood, R.: The effect of solar zenith angle on MODIS cloud optical and microphysical retrievals within marine liquid water clouds, Atmos. Chem. Phys., 14, 7291-7321, doi:10.5194/acp-14-7291-2014, 2014.

Gueymard, C. A.: The sun's total and spectral irradiance for solar energy applications and solar radiation models, Sol. Energy, 76, 423-453, 2004.

Intrieri, J. M., Fairall, C. W., Shupe, M. D., Persson, P. O. G., Andreas, E. L., Guest, P. S., and Moritz, R. E.: An annual cycle of Arctic surface cloud forcing at SHEBA, J. Geophys. Res., 107, SHE 13-1-SHE 13-14, doi:10.1029/2000JC000439, 2002a.

Intrieri, J. M., Shupe, M. D., Uttal, T., and McCarty, B. J.: An annual cycle of Arctic cloud characteristics observed by radar and lidar at SHEBA, J. Geophys. Res., 107, SHE 5-1-SHE 5-15, doi:10.1029/2000JC000423, 2002b.

Iwabuchi, H.: Efficient Monte Carlo methods for radiative transfer modeling, J. Atmos. Sci., 63, 2324-2339, 2006.

Iwabuchi, H. and Kobayashi, H.: Modeling of radiative transfer in cloudy atmospheres and plant canopies using Monte Carlo methods, Tech. Rep. 8, 199 pp., FRCGC, 2008.

Jäkel, E., Wendisch, M., and Mayer, B.: Influence of spatial heterogeneity of local surface albedo on the area-averaged surface albedo retrieved from airborne irradiance measurements, Atmos. Meas. Tech., 6, 527-537, doi:10.5194/amt-6-527-2013, 2013.

King, N. J., and Vaughan, G.: Using passive remote sensing to retrieve the vertical variation of cloud droplet size in marine stratocumulus: An assessment of information content and the potential for improved retrievals from hyperspectral measurements, J. Geophys. Res., 117, D15206, doi:10.1029/2012JD017896, 2012.

Klingebiel, M., de Lozar, A., Molleker, S., Weigel, R., Roth, A., Schmidt, L., Mayer, J., Ehrlich, A., Neuber, R., Wendisch, M., and Borrmann, S.: Arctic low-level boundary layer clouds: in situ measurements and simulations of mono- and bimodal supercooled droplet size distributions at the top layer of liquid phase clouds, Atmos. Chem. Phys., 15, 617-631, doi:10.5194/acp-15617-2015, 2015.

Krijger, J. M., Tol, P., Istomina, L. G., Schlundt, C., Schrijver, H., and Aben, I.: Improved identification of clouds and ice/snow covered surfaces in SCIAMACHY observations, Atmos. Meas. Technol., 4, 2213-2224, doi:10.5194/amt-4-2213-2011, 2011.

Lawson, R. P., Baker, B. A., and Schmitt, C. G.: An overview of microphysical properties of Arctic clouds observed in May and July 1998 during FIRE ACE, J. Geophys. Res., 106, 14989-15014, 2001.

Lindsay, R. W. and Rothrock, D. A.: Arctic sea-ice albedo from Avhrr, J. Climate, 7, 1737-1749, doi: 10.1175/15200442(1994)007<1737:ASIAFA>2.0.CO;2, 1994.

Loeb, N. G. and Davis, R.: Observational evidence of plane parallel model biases: Apparent dependence of cloud optical depth on solar zenith angle, J. Geophys. Res., 101, 1621-1634, 1996.

Lyapustin, A.: Three-dimensional effects in the remote sensing of surface albedo, IEEE T. Geosci. Remote, 39, 254-263, 2001.

Lyapustin, A. and Kaufman, Y.: Role of adjacency effect in the remote sensing of aerosol, J. Geophys. Res., 106, 11909-11916, 2001.

Marshak, A., Wen, G., Coakley Jr., J. A., Remer, L. A., Loeb, N. G., and Cahalan, R. F.: A simple model for the cloud adjacency effect and the apparent bluing of aerosols near clouds, J. Geophys. Res., 113, D14S17, doi:10.1029/2007JD009196, 2008.

Nakajima, T. and King, M.: Determination of the optical thickness and effective particle radius of clouds from reflected solar radiation measurements. Part I: Theory, J. Atmos. Sci., 47, 18781893, 1990.

Overland, J. E., Wood, K. R., and Wang, M.: Warm Arctic-cold continents: impacts of the newly open Arctic Sea, Polar Res., 30, 15787, doi:10.3402/polar.v30i0.15787, 2011.

Pierluissi, J. and Peng, G.-S.: New molecular transmission band models for LOWTRAN, Opt. Eng., 24, 541-547, 1985.

Platnick, S. and King, M. D.: The MODIS Cloud Products: Algorithms and Examples From Terra, IEEE T. Geosci. Remote. 41, 459-473, 2003.

Platnick, S., Li, J. Y., King, M. D., Gerber, H., and Hobbs, P. V.: A solar reflectance method for retrieving the optical thickness and droplet size of liquid water clouds over snow and ice surfaces, J. Geophys. Res., 106, 15185-15199, 2001.

Platnick, S., King, M. D., Wind, B., Gray, M., and Hubanks, P.: An initial analysis of pixel-level uncertainty in global MODIS cloud optical thickness and effective particle size retrievals, Proc. SPIE, 30, 5652, doi:10.1117/12.578353, 2004.

Ricchiazzi, P. and Gautier, C.: Investigation of the effect of surface heterogeneity and topography on the radiation environment of Palmer Station, Antarctica, with a hybrid 3-D radiative transfer model, J. Geophys. Res., 103, 6161-6178, 1998.

Rothrock, D. A. and Thorndike, A. S.: Measuring the sea ice floe size distribution, J. Geophys. Res., 89, 6477-6486, 1984.

Sanderson, M. G., Hemming, D. L., and Betts, R. A.: Regional temperature and precipitation changes under high-end $(\geq 4$ degrees C) global warming, Philos. T. R. Soc. A., 369, 85-98, doi:10.1098/rsta.2010.0283, 2011.

Schäfer, M., Bierwirth, E., Ehrlich, A., Heyner, F., and Wendisch, M.: Retrieval of cirrus optical thickness and assessment of ice crystal shape from ground-based imaging spectrometry, Atmos. Meas. Tech., 6, 1855-1868, doi:10.5194/amt-61855-2013, 2013.

Shupe, M. D., Walden, V. P., Eloranta, E., Uttal, T., Campbell, J. R., Starkweather, S. M., and Shiobara, M.: Clouds at Arctic atmospheric observatories. Part I: Occurrence and macrophysical properties, J. Appl. Meteorol. Clim., 50, 626-644, doi:10.1175/2010JAMC2467.1, 2011.

Stachlewska, I. S., Neuber, R., Lampert, A., Ritter, C., and Wehrle, G.: AMALi - the Airborne Mobile Aerosol Lidar for Arctic research, Atmos. Chem. Phys., 10, 2947-2963, doi:10.5194/acp-10-2947-2010, 2010.

Tsay, S.-C. and Jayaweera, K.: Physical Characteristics of Arctic Stratus Clouds, J. Clim. Appl. Meteorol., 23, 584-596, 2013.

Vavrus, S.: The Impact of Cloud Feedbacks on Arctic Climate under Greenhouse Forcing, J. Climate., 17, 603-615, 2004.

Wendisch, M. and Brenguier, J.-L.: Airborne Measurements for Environmental Research - Methods and Instruments, Wiley-VCH Verlag GmbH \& Co. KGaA, Weinheim, Germany, 2013.

Wendisch, M., Müller, D., Schell, D., and Heintzenberg, J.: An airborne spectral albedometer with active horizontal stabilization, J. Atmos. Ocean. Tech., 18, 1856-1866, 2001.

Wendisch, M., Yang, P., and Ehrlich, A., (Eds.): Amplified climate changes in the Arctic: Role of clouds and atmospheric radiation, vol. 132, 1-34, Sitzungsberichte der 
Sächsischen Akademie der Wissenschaften zu Leipzig, Mathematisch-Naturwissenschaftliche Klasse, S. Hirzel Verlag, Stuttgart/Leipzig, 2013.

Werner, F., Siebert, H., Pilewskie, P., Schmeissner, T., Shaw, R. A., and Wendisch, M.: New airborne retrieval approach for trade wind cumulus properties under overlying cirrus, J. Geophys. Res., 118, 1-16, doi:10.1002/jgrd.50334, 2013.
Zinner, T., Wind, G., Platnick, S., and Ackerman, A. S.: Testing remote sensing on artificial observations: impact of drizzle and 3-D cloud structure on effective radius retrievals, Atmos. Chem. Phys., 10, 9535-9549, doi:10.5194/acp-10-9535-2010, 2010. 